\title{
Spontaneous traveling waves naturally emerge from horizontal fiber time delays and travel through locally asynchronous-irregular states
}

\section{Zachary Davis ( $\sim$ zdavis@salk.edu )}

Salk Institute for Biological Studies https://orcid.org/0000-0003-4440-9011

Gabriel Benigno

Western University

Charlee Fletterman

Salk Institute for Biological Studies

Theo Desbordes

Salk Institute for Biological Studies

\section{Terrence Sejnowski}

Salk Institute for Biological Studies https://orcid.org/0000-0002-0622-7391

John Reynolds

The Salk Institute for Biological Studies

Lyle Muller

Western University

\section{Article}

Keywords: sensory neuroscience, spontaneous fluctuations, cortical fibers

Posted Date: December 2nd, 2020

DOl: https://doi.org/10.21203/rs.3.rs-113978/v1

License: (c) (i) This work is licensed under a Creative Commons Attribution 4.0 International License.

Read Full License

Version of Record: A version of this preprint was published at Nature Communications on October 18th, 2021. See the published version at https://doi.org/10.1038/s41467-021-26175-1. 


\section{Spontaneous traveling waves naturally emerge from horizontal fiber time delays and} travel through locally asynchronous-irregular states

3 5 6 7

Authors: Zachary W. Davis ${ }^{1, *}$, Gabriel B. Benigno ${ }^{2,3,{ }^{*},}$, Charlee Fletterman ${ }^{1,{ }^{*}}$, Theo Desbordes ${ }^{1}$, Terrence J. Sejnowski ${ }^{1, \dagger}$, John Reynolds ${ }^{1, \dagger}$, Lyle Muller ${ }^{2,3, \dagger}$

\section{Affiliations:}

${ }^{1}$ The Salk Institute for Biological Studies, La Jolla, CA, USA.

${ }^{2}$ Department of Applied Mathematics, Western University, London, ON, Canada. ${ }^{3}$ Brain and Mind Institute, Western University, London, ON, Canada.

${ }^{*}$ Authors contributed equally, ${ }^{\dagger}$ Co-senior authors

* Corresponding authors J.H.R. and L.M., emails: reynolds@salk.edu and Imuller2@uwo.ca

\section{Abstract}

Sensory neuroscience has focused a great deal of its attention on characterizing the mean firing rate that is evoked by a stimulus, and while it has long been recognized that the firing rates of individual neurons fluctuate around the mean, these fluctuations are often treated as a form of internally generated noise ${ }^{1}$. There is, however, evidence that these "ongoing" fluctuations of activity in sensory cortex during normal, waking function shape neuronal excitability and responses to external input ${ }^{2,3}$. We have recently found that spontaneous fluctuations are organized into waves traveling at speeds consistent with the speed of action potentials traversing unmyelinated horizontal cortical fibers $(0.1-0.6 \mathrm{~m} / \mathrm{s})^{4}$ across the cortical surface ${ }^{5}$. These waves systematically modulate excitability across the retinotopic map, strongly affecting perceptual sensitivity as measured in a visual detection task. The underlying mechanism for these waves, however, is unknown. Further, it is unclear whether waves are consistent with the low rate, highly irregular, and weakly correlated "asynchronous-irregular" dynamics observed in computational models ${ }^{6}$ and cortical recordings in vivo ${ }^{7}$. Here, we study a large-scale computational model of a cortical sheet, with connections ranging up to biological scales. Using an efficient custom simulation framework, we study networks with topographically-organized connectivity and distance-dependent axonal conduction delays from several thousand up to one million neurons. We find that spontaneous traveling waves are a general property of these networks and are consistent with the asynchronous-irregular regime. These waves are well matched to spontaneous waves recorded in the neocortex of awake monkeys. Further, individual neurons 
sparsely participate in waves, yielding a sparse-wave regime that offers a unique operating mode, where traveling waves coexist with locally asynchronous-irregular dynamics, without inducing deleterious neuronal correlations ${ }^{8}$.

\section{Introduction}

Visual cortical neurons exhibit variable fluctuations in their spontaneous activity and stimulusevoked responses. Rather than being due to noise intrinsic to the neural spiking mechanism ${ }^{9}$, which is highly reliable ${ }^{10}$, variability is thought to emerge from ongoing synaptic activity in the dense recurrent connectivity of cortical networks ${ }^{1,2}$. When observed from a single point in the cortex, spontaneous fluctuations resemble a broadband temporal noise process ${ }^{1,11}$. Multisite recordings have revealed that these temporal fluctuations can be part of waves traveling across a cortical area ${ }^{12-16}$. Spontaneous traveling waves had largely been observed in slow-wave fluctuations associated with anesthesia, sleep, or low arousal. While traveling waves had been theorized to have an impact on cortical computation, it was difficult to identify their role since active cortical states exhibit fluctuations that are more complex, dominated by higher-frequency and lower-amplitude activity ${ }^{17}$, making these waves harder to detect. Further, driving input is believed to quench variability in ongoing dynamics, ${ }^{18}$ calling into question the potential impact of traveling waves on evoked activity ${ }^{19}$.

Recent work has shown that spontaneous traveling waves are present in the awake state, that they influence the magnitude of sensory-evoked activity, and that-depending on their retinotopic alignment with sensory input-they can improve perceptual sensitivity ${ }^{5}$. However, the mechanisms that generate them, and whether they are consistent with the asynchronousirregular spiking dynamics characteristic of awake cortex ${ }^{20}$, is unknown. Based on their speed of propagation, we hypothesize these waves result from action potentials propagating along unmyelinated horizontal fibers. To test this hypothesis, we studied a spiking network model across a range of biologically realistic neuronal densities, distance-dependent connection probabilities, excitatory/inhibitory balances, and synaptic conductance states. Importantly, this model incorporated axonal time delays from conduction along unmyelinated horizontal fibers, which shaped ongoing activity patterns into traveling waves consistent with those observed in vivo. Spontaneous traveling waves were apparent in this network model and occurred consistently across a wide parameter range that produced asynchronous-irregular dynamics. 
One might wonder whether the occurrence of these traveling waves induces correlated variability, which has been found to impair perception ${ }^{8}$. Results from the spiking network model show this need not be the case. In both the computational model and multielectrode recordings in the marmoset visual system, we found neuronal participation in these waves was sparse, meaning that the probability of any individual neuron contributing to an individual traveling wave was low. We thus refer to the model as the sparse-wave model and this regime as the sparsewave regime. Therefore, spontaneous waves can emerge at mesoscopic scales in subthreshold synaptic fluctuations, carried by sparse spiking activity along horizontal fibers, without inducing changes in pairwise correlations in the activity of individual neurons. Traveling waves can thus co-exist with a locally asynchronous-irregular state, conferring their benefits while maintaining the computational advantages of this dynamical regime ${ }^{7,21}$.

\section{Results}

Spontaneous synaptic fluctuations are comparable to those during stimulus-evoked responses

Previous work has shown that moment-by-moment fluctuations in synaptic input in the cortex can be on the same order of magnitude as during the sustained period of stimulus-evoked responses ${ }^{12,22-24}$. Fluctuating synaptic inputs can have a significant impact on neural excitability ${ }^{25}$, gain modulation ${ }^{26}$, and readout of sensory information ${ }^{13}$. To understand the impact of the spontaneous network state on evoked responses in the awake visual cortex, we recorded spontaneous and stimulus-evoked activity from chronically implanted multi-electrode Utah arrays (Blackrock Microsystems) in area MT of two common marmosets (Callithrix jacchus; data previously reported in Davis et al., 2020). Spontaneous multiunit activity recorded from a single electrode while a marmoset fixated a fixation point was characterized by a low, irregular firing rate. The appearance of a highly salient stimulus (10\% Michelson contrast drifting Gabor) within the multiunit receptive field evoked a robust response (Figure 1a). When measured over many repeated presentations of the stimulus, the mean multiunit firing rate rose from $13 \pm 1.6 \mathrm{sp} / \mathrm{sec}$ during fixation, to $97 \pm 5.7 \mathrm{sp} / \mathrm{sec}$ in response to the stimulus ( $N=40$ trials over 3 recording sessions). These evoked spiking responses were variable from trial to trial (mean fano factor $=$ $1.01 \pm 0.01 \mathrm{SEM}, 40$ to $240 \mathrm{~ms}$ after stimulus onset), consistent with previous observations ${ }^{18,27,28}$.

This variability is partly the result of ongoing spontaneous fluctuations in synaptic activity in the local population at the time of the evoked spiking response ${ }^{12,13}$. These fluctuating synaptic inputs, in turn, contribute to the local field potential (LFP) ${ }^{29,30}$. When averaged across high-contrast trials, 
the LFP had a robust negative deflection aligned to the stimulus-evoked spiking response, while the pre-stimulus period was flat (black line, Figure 1a). However, at the single-trial level, the stimulus-evoked LFP response was similar in magnitude to the spontaneous fluctuations occurring during fixation (right panel, Figure 1a). The relative power between the LFP just prior to the stimulus (-200 to $0 \mathrm{~ms})$ and following stimulus onset $(0$ to $+200 \mathrm{~ms})$ across single trials had a small but significant difference from $0 \mathrm{~dB}$, which represents parity between spontaneous and stimulus-evoked fluctuations (median $1.98 \mathrm{~dB}, \mathrm{p}<0.001$ Wilcoxon signed-rank test).

While strong, high-contrast visual stimulation evoked slightly stronger LFP fluctuations than intrinsic network fluctuations, the distinction disappears in the context of weak visual inputs (Figure 1b). When the marmoset was presented a faint stimulus that was detected approximately $50 \%$ of the time ( $<2 \%$ Michelson contrast), the evoked spiking response was significantly weaker and more variable (mean $=68 \pm 4.4 \mathrm{sp} / \mathrm{sec}, \mathrm{p}<0.001$; fano factor $=1.55 \pm 0.09, \mathrm{p}<0.01$, Wilcoxon rank-sum test). This corresponded to a weaker average LFP response, and the trialby-trial relative power between spontaneous and evoked fluctuations was not significantly different from $0 \mathrm{~dB}$ (median $=0.18 \mathrm{~dB}, \mathrm{p}=0.55$ Wilcoxon ranked-sum test).

Given the relative magnitude of spontaneous fluctuations to responses evoked by weak sensory inputs, we hypothesized that much of the variability in neuronal spiking responses could be explained by the state of the local network. We recently reported that spontaneous LFP fluctuations in awake cortex are organized into waves that travel across an entire cortical area (Figure 1c). They modulate spontaneous spiking probability (Figure 1d), and they directly impact the magnitude of stimulus-evoked responses depending on their alignment with neuronal receptive fields (Figure 1e). We found that, rather than acting as a source of noise that impairs perception, spontaneous waves can-depending on their spatiotemporal alignment with a visual stimulus-improve the monkeys' ability to detect the stimulus. We thus sought to understand what mechanisms might generate traveling waves in cortex and test whether they represent an operating regime either consistent with or distinct from the irregular, asynchronous activity patterns classically observed in silico $0^{6,31}$ and in vivo ${ }^{7,32}$.

Spontaneous traveling waves can emerge in network models without altering individual neuron spiking statistics 
To address this question, we studied large-scale spiking network models composed of leaky integrate-and-fire neurons with balanced excitation and inhibition and conductance-based synapses. When neuronal interactions are modeled as conductances, taking into account the time-dependent driving forces and channel activations at the synapse, spiking network models can enter into states of self-sustained activity ${ }^{33,34}$. Asynchronous-irregular activity ${ }^{6}$ in these selfsustained states, generated without external drive, results naturally from the recurrently generated fluctuations intrinsic to the dynamics of the system ${ }^{33,34}$. These dynamics are characterized by low, variable firing rates, weak pairwise correlations, and coefficients of variation (C.V.) near unity. These self-sustained states provide an opportunity to study spiking network dynamics that are structured by the recurrent activity of the network itself, rather than dominated by random external Poisson synaptic input ${ }^{21}$, and are well suited to model the spontaneous background activity observed in cortex during active perception.

We first studied a two-dimensional conductance-based spiking network model with over $1,000,000$ neurons distributed over a $6 \times 6 \mathrm{~mm}$ area consisting of 80 percent excitatory and 20 percent inhibitory neurons, randomly connected with 3,000 synapses per cell, yielding a sparsely connected network. We eliminated the outer milimeter from analysis, yielding a $4 \times 4 \mathrm{~mm}$ area with 450,000 neurons. These values were selected to approximate the density and connectivity of neurons in cortical layer $2 / 3$ of area MT in the common marmoset ${ }^{35,36}$. This randomlyconnected network generated self-sustained activity with spontaneous spiking fluctuations consistent with the asynchronous-irregular regime $e^{6,37}$ and lacked any spatiotemporal structure (Fig. 2b). A simulated LFP was calculated from summed excitatory and inhibitory synaptic activity over $10 \times 10$ adjacent, non-overlapping pools of neurons (corresponding to $67.8 \times 67.8 \mu \mathrm{M}$ ) ${ }^{38}$ and was used to estimate the local excitability state at each point in the network for comparison to the electrophysiological recordings. The LFP was homogeneous across the network, as would be expected from pools of neurons receiving synaptic input from random positions in the network (Fig. 2c).

To test whether topographic connections with transmission delays were sufficient to generate spontaneous traveling waves in the network, as a refinement to the model described in Figure 2a, two key elements were introduced: 1) connection probability decayed as a Gaussian with distance between neurons $(\sigma=400 \mu \mathrm{M})^{39,40}$ to mimic the topographic connectivity in cortex and 2) action potentials activated synaptic currents after a time delay determined by the distance of the connection to simulate the conduction velocity of horizontal fibers in cortex $\left(v_{c}=0.2 \mathrm{~m} / \mathrm{s},{ }^{4}\right.$ 
Fig. 2d). This network also produced self-sustained, spontaneous fluctuations, but spiking activity was sparsely organized into bands that moved across the network as waves (rasters, Fig. 2e). LFP fluctuations were heterogeneous across the network and exhibited organized spatial structure with localized regions coordinated in amplitude (Fig. 2f).

To test whether the presence of these organized topographic fluctuations altered the asynchronous-irregular dynamics of individual neurons in the network, we compared the firing rates and C.V. across a randomly selected population of excitatory neurons $(N=5000)$. There was no difference in the distribution of firing rates across the networks $(p=0.68$, KolmogorovSmirnov test; Fig. $2 g$ ) or in the distribution of C.V. ( $p=0.79$, Kolmogorov-Smirnov test; Fig. $2 h$ ). Therefore, individual neurons maintained their asynchronous and irregular firing states while the topographically-connected network produced spontaneous traveling waves.

While one might expect the organized bands of spiking activity would result in increased correlations across neurons, we found no evidence that this was the case. The introduction of topographic connections did not affect pairwise correlations, as the degree of spike-spike coherence between the randomly- and topographically-connected networks was indistinguishable $(\mathrm{N}=1000$ random pairs; $\mathrm{Cl}$ test, $\mathrm{a}=0.05$; Fig. 2i). Spatiotemporal structure can exist in these networks without disrupting C.V. or pairwise coherence because spikes are sparsely organized into waves, with only a small fraction of neurons spiking in any given wave. We therefore refer to this as the "sparse-wave" network regime. If it were the case that neurons strongly participated in these fluctuations, then they would show a degree of coherence in the range of frequencies dominated by those fluctuations. To demonstrate this, we simulated a smaller network with fewer neurons and denser connections which generated spontaneous fluctuations that strongly regulated spiking activity. This "dense-wave" network did exhibit strong spike-spike coherence in the frequency band dominated by fluctuations in the LFP (Fig. S1, model parameters table S1). Therefore, unlike in the dense-wave network, traveling waves in the sparse-wave regime do not necessarily induce pairwise correlation across the network.

As hypothesized, the addition of topographic connections and conduction delays was sufficient to produce clear spatiotemporal organization in the network activity (Fig. 3a). To classify these activity patterns as traveling waves and quantify their properties relative to cortical recordings from marmoset cortex, we applied the same analysis technique developed for the experimental recordings (Generalized Phase, GP5) to the simulated LFP in each $10 \times 10$ neuronal pool. We 
then adapted a technique previously developed for detecting traveling waves in noisy multielectrode recordings ${ }^{41,42}$. We estimated the gradient of phase at each moment in time and calculated putative wavelengths and propagation speeds (Fig. $3 b$ and d). We then identified places and times in the network where the organization of activity into traveling waves was significant. Significance was determined by comparing the observed wavelengths to the wavelength distribution after a spatial shuffle of electrode positions, with the 99th percentile of the shuffle distribution taken as the threshold criterion (Fig. 3c-d). This approach provides a sensitive and robust means to detect traveling waves from moment to moment ${ }^{41,42}$. We found significant wave activity in the topographically-connected network approximately 50 percent of the time, whereas the presence of significantly structured wave organization was absent from networks with random connections and no delays (Fig. 3e) as the distribution of putative wavelengths was similar to the shuffled distribution (Fig. 3f).

\section{Spontaneous waves occur throughout the asynchronous-irregular regime}

In the example network, topographic connections were sufficient to induce large-scale waves of activity without disrupting the fine scale asynchronous-irregular dynamics of individual neurons. Does the presence of traveling waves generalize across all networks with asynchronousirregular activity, topographic connections, and axonal conduction delays ${ }^{34,37}$ ? We scanned across 2,500 combinations of different excitatory and inhibitory (E/I) conductances in the topographically-connected model and found 654 combinations that produced self-sustained spiking activity. We then identified networks with asynchronous-irregular spiking dynamics, defined as networks with mean excitatory firing rates between 1 and $25 \mathrm{sp} / \mathrm{sec}$, mean C.V. between 0.7 and 1.4 , and mean pairwise correlations between -0.1 and $0.1^{37}$. Approximately 98 percent (646 out of 654) of the self-sustained networks were classified as asynchronousirregular. We then measured the percentage of time each network's activity was significantly organized into traveling waves. Waves were present across the entire range of asynchronousirregular networks (Fig. 4a). The strength of wave activity was negatively correlated with the magnitude of $E / l$ conductance (Pearsons's $r=-0.35 \pm 0.07,95 \% \mathrm{Cl}$; Fig. 4a) indicating weaker synapses led to stronger wave activity. The average wavelength was also negatively correlated with synaptic strength (Pearsons's $r=-0.41 \pm 0.07$; Fig. 4b), with weaker excitatory conductances particularly leading to longer wavelengths (Pearson's $r=-0.61 \pm 0.05$ for $g_{e}$ vs. $r=-0.21 \pm 0.07$ for $g_{i}$ ). These results demonstrate that spontaneous traveling waves are a general property of topographic connectivity and are entirely consistent with locally asynchronous-irregular states. 
238 How important is network scale in generating traveling waves? To answer this question, we

239 simulated networks ranging from $0.5 \mathrm{~mm}$ to $4 \mathrm{~mm}$, holding neuronal and connection density 240 constant. For small networks $(\sim 0.5 \mathrm{~mm})$, a very limited range of the $E / /$ space produced self241 sustained and asynchronous network dynamics. As network size grew, the asynchronous242 irregular parameter space grew as well, extending to include smaller and smaller combinations 243 of E/l synaptic strength ${ }^{20}$ (Fig. 4c). It was thus necessary to simulate spiking network models at 244 sufficient spatial scales $(>1 \mathrm{~mm})$ to generate asynchronous-irregular activity in networks with 245 conductances resembling those estimated in vivo ${ }^{20}$. At small network scales, wavelength 246 distributions during asynchronous-irregular dynamics were not distinct from the spatial shuffle, and the parameters that favored longer wavelengths did not produce asynchronous-irregular activity. Only at larger network scales did wave activity become strongly apparent (Fig. 4d).

Network connectivity determines wave properties

What effect did our chosen parameters for connection distance and conduction velocity have on wave properties? We hypothesized the spatial extent of connections and the conduction speed of spikes directly control the wavelength and propagation speed, respectively, of traveling waves in the model. To test these predictions we simulated networks with various values of standard deviation $\left(\sigma_{s}\right)$ for the Gaussian connection probability distribution. Consistent with our hypotheses, the distribution of wavelengths increased with larger connection distances (Fig. 5a) and increasing the conduction velocity led to increased average wave speeds (Fig. 5b). Thus, the macroscopic features of spontaneous traveling waves were directly related to specific network structures in the model.

Are these waves only possible with perfectly Gaussian connection profiles and uniform conduction velocity, or can they tolerate a broad range of values similar to those observed in vivo? To test this, we simulated the example model in Figure 2, with 10 percent of the connections randomly rewired with uniform probability across the network, generating long range connections $^{43,44}$. The conduction velocity along each connection was drawn from the range of 267 conduction speeds observed for unmyelinated horizontal fibers in cortex $\left(0.1\right.$ to $\left.0.6 \mathrm{~m} / \mathrm{s}^{4,45,46}\right)$. 268 Spontaneous traveling waves persisted under these network conditions (Fig. 5c, d), indicating 269 the presence of waves was not limited to a fixed or limited set of homogeneous network 270 properties, but instead also occurred in networks with large heterogeneity, as in cortex. 
274 How well do the dynamics observed in our simulations match the dynamics observed in 275 electrophysiological recordings of the cortex? To test this we compared the model results to the 276 data recorded from marmoset MT while monkeys fixated a spot at the center of an otherwise 277 grey computer screen. We measured the mean firing rates (Fig. 6a) and C.V. (Fig. 6b) across 278 the population of single- and multi-unit activity over multiple recording sessions. The distributions of firing rates and C.V. were qualitatively similar between the recorded data and the sparse-wave model, suggesting the spontaneous dynamics in the cortical recordings are also consistent with the asynchronous-irregular regime.

We next measured the distribution of estimated wavelengths in the data and compared this to the wavelength distribution in the model. LFP data in the cortex is not independent across electrodes (as it is in our simulation), but rather pools signals from a cortical volume of approximately $250 \mu \mathrm{M}$ in radius around the electrode tip ${ }^{29,30}$ and has correlations that fall off with distance $^{17}$. To emulate these properties of cortical LFP recordings, we applied a smoothing kernel that expanded the area of integration from each simulated LFP point and reduced the independence of the signal. After smoothing our simulated LFP and quantifying wave properties, the distribution of simulated wavelengths closely approximated the distribution observed in cortex (dotted gray and red lines, Fig. 6c). Similarly, the distribution of observed speeds in both the network simulation and the data covered the range of conduction velocities in the horizontal fibers, peaking at approximately $0.2 \mathrm{~m} / \mathrm{s}$ (Fig. 6d). Thus, across four different measures (spikerate, spike-variability, wave-size, wave-velocity), the distributions characterizing activity in the network model were in close alignment with experimental recordings.

Neurons sparsely participate in waves due to weak coupling to synaptic fluctuations

What is the conductance state of spontaneous fluctuations in the sparse-wave network simulation? Membrane potential fluctuations were consistent with high conductance states, with a Gaussian distribution whose mean was close to the spiking threshold (black line, Fig. 7a). This contrasted with the skewed distribution of membrane potentials in the dense wave network, which was consistent with a low conductance state transitioning between quiescenthyperpolarized and bursting-depolarized membrane potentials (purple line, Fig. 7a). In the 
sparse-wave network model, stochastic fluctuations in the membrane potential produced sparse and irregular spiking activity. These fluctuations were driven by shifts in excitatory-inhibitory balance across the local population which, due to the topographic network connections, were shared by adjacent populations and carried by spikes across horizontal connections. These summed currents in our estimate of the LFP exhibited a counterphase relationship with the relative $E / I$ balance: the inhibition-dominated $E / I$ regime produced positive LFP potentials, and the excitation-dominated E/I regime produced negative LFP potentials (Fig. 7b, c).

To demonstrate that simulated neurons are sparsely modulated by traveling waves, we measured the LFP phase at which each spike occurred (10 bins from $-\pi$ to $\pi$; $z$-scored to normalize across variable firing rates in each network; Fig. 7d) for $E / I$ conductances that yielded asynchronous-irregular dynamics. The degree of spike-phase modulation was significant across the entire parameter space, with spikes more likely during phases closer to $\pm \pi$. The magnitude of this modulation was inversely correlated with the magnitude of $E / /$ conductances (Pearson's $r$ $=-0.44 \pm 0.09,95 \% \mathrm{Cl} ; \mathrm{N}=646$ simulations), with the largest modulations in the range of the smallest conductance values that supported asynchronous-irregular activity in our simulations (where traveling waves were most frequent and also had the longest wavelengths; Fig. 4a and $4 b$, respectively). We chose a point among these small conductance values ( $1 \mathrm{nS} \mathrm{g}_{\mathrm{e}}, 10 \mathrm{nS} \mathrm{g}_{\mathrm{i}}$ ), to compare the degree of coupling between the model and the cortical recordings. There was no difference between the spike-phase modulation observed in the sparse-wave network model and the recorded data $(\mathrm{N}=22$ matched resamplings; model circular-resultant $=0.15 \pm 0.001$ S.E.M.; data circular-resultant $=0.16 \pm 0.005 ; p=0.28$, Wilcoxon signed-rank test), although the preferred phase-angle differed slightly between the data and model (data-preferred phase $=3.05$ rad, model-preferred phase $=-1.96 \mathrm{rad}$ ).

While there was a similar degree of spike-phase modulation between the cortical data and the sparse-wave model, the modulation was significantly stronger in the dense-wave model $(\mathrm{N}=10$ resamples; circular-resultant $=0.43 \pm 0.006, p<0.00001$, Wilcoxon rank-sum test; Fig. $7 g)$. The phase distribution follows this pattern as well, with both the cortical data and sparse-wave model spiking more near $\pm \pi$ phases, whereas the dense-wave model spiking is minimal at these phases. Additionally, the randomly-connected network showed the weakest spike-phase relationship ( $\mathrm{N}=22$ resamples; circular-resultant $=0.07 \pm 0.001 ; \mathrm{p}<0.0001$ Wilcoxon signedrank test; Fig. S2), as expected from a network where the neurons in the LFP pool draw from inputs distributed throughout the entire network. These results demonstrate that-in the 
simulated large-scale spiking networks-waves exist primarily in subthreshold synaptic fluctuations that are carried through sparse, phase-modulated spiking activity traveling along topographically distributed horizontal fibers. The presence of a similar spike-phase relationship in vivo, particularly for model conductance states which corresponded to experimental estimates of neuronal conductance states ${ }^{20}$, demonstrates that the sparse-wave regime in the model is consistent with the properties of waves observed in the experimental recordings.

\section{Spontaneous traveling waves modulate responses to inputs}

Finally, we hypothesized the state of network fluctuations in the sparse-wave network model would modulate the magnitude of responses evoked by feed-foward inputs. To test this, we stimulated one $10 \times 10$ neuron pool in the sparse-wave network with a $50 \mathrm{~Hz}$ Poisson spike train on 100 afferent synapses to each neuron to mimic feed-forward stimulus input to the network. We stimulated for $10 \mathrm{~ms}$, aligned either to the depolarized or hyperpolarized state of network fluctuations defined, respectively, by the most and least probable phases for spikes to occur according to the network's spontaneous spike-phase distribution. When spiking inputs were aligned to the depolarized state, the evoked spiking responses were boosted (blue lines, Fig. 8a) relative to weaker responses when inputs were aligned to the hyperpolarized state (red lines, Fig. 8a). These effects were consistent with wave-modulated visual responses to motion stimuli observed in area MT in vivo (cf. Fig. 1e). In contrast, stimulation in the depolarized state was wholly suppressed in the smaller, dense-wave network and the hyperpolarized state yielded higher responses (red and blue lines, Fig. 8b).

To quantify the effect of the traveling waves on evoked responses in each network, we then calculated the gain modulation, which is the ratio between the maximum stimulus-evoked response and the spontaneous spiking rate when no stimulus was applied (Fig. 8c). Across repeated input stimulations, input gain was much stronger for the depolarized state relative to the hyperpolarized state in the sparse-wave network $(N=20$ stimulations; depolarized state = $4.25 \pm 0.11$; hyperpolarized state $=1.74 \pm 0.08$ ). In contrast, in the dense-wave network the strong spontaneous fluctuations shunted the incoming spikes, resulting in larger gain for the hyperpolarized state relative to the depolarized state (depolarized state $=1.11 \pm 0.01$;

370 hyperpolarized state $=1.74 \pm 0.07$ ). The highest gain in the dense-wave model was significantly 371 weaker than the depolarized gain in the sparse-wave network $\left(p<1 \times 10^{-7}\right.$, Wilcoxon ranked-sum 372 test). The increase in gain that occurs in the sparse-wave network mirrors our observations of 
wave-dependent sensitivity in awake monkeys performing a threshold detection task ${ }^{5}$. Thus the sparse-wave model offers a mechanistic account for observed phase-dependent modulations of weak sensory responses by traveling waves measured in vivo that a network characterized by dense-wave dynamics fails to replicate.

\section{Discussion}

The present work builds on and seeks to explain our recent finding that spontaneous fluctuations in cortical activity modulate the moment-to-moment processing of sensory information in a manner that affects perceptual sensitivity. These fluctuations are neither synchronous across the cortical surface nor independent noise processes. Rather, they are structured as traveling waves. The model presented here shows that distance-dependent conduction delays in topographic, conductance-based spiking network models are sufficient to account for our results in vivo. Waves occur spontaneously, without requiring a driving input, and they occur robustly, in the sense that they are generated across a wide parameter space and in the sense that they occur across the entire space of $\mathrm{E} / \mathrm{l}$ conductances that gives rise to asynchronous-irregular activity dynamics. The properties of these waves depend systematically on the scales of distance-dependant connections and the speeds of action-potential propagation. The waves were well matched to those observed in cortical recordings from behaving marmosets ${ }^{5}$ for speeds consistent with the conduction velocity of unmyelinated horizontal fibers. Neurons sparsely participated in these waves at the scales of neuronal density and connectivity found in cortex. The sparseness of the waves allowed them to occur without disturbing the asynchronousirregular dynamics that are observed in cortical activity and have advantages for neural computation $2,7,21,47,48$. These sparse-wave networks remain sensitive to spiking inputs, producing evoked responses modulated in a phase-dependent manner, as observed in vivo. This is in contrast to smaller scale networks that exhibit dense-waves that drive correlated fluctuations across the population and render the network insensitive to spiking inputs.

These results demonstrate the importance of considering distance-dependent time delays in neural systems. When considered at the scales of entire cortical areas, individual horizontal fibers can span distances ranging from hundreds of microns to several millimeters ${ }^{43,44}$, with axonal conduction delays on the order of tens of milliseconds ${ }^{4,45}$. While previous computational models held that the contribution of axonal delays was negligible in their effects on the temporal properties of spiking networks ${ }^{49}$, and they are rarely considered, others have found delays to be 
important in shaping neural dynamics ${ }^{50,51}$. Traveling waves have been described in topographic spiking network models that lack distance-dependent delays, but only in restricted parameter ranges ${ }^{52-54}$. With distance-dependent delays, however, we find that traveling waves occur across the whole asynchronous-irregular regime, in models that are sufficiently large in scale. Thus, not only are spontaneous traveling waves consistent with the asynchronous-irregular regime, they are a necessary consequence of topographic connectivity and time delays in cortex across conditions that yield asynchronous-irregular spiking.

Critically, the waves we observe sparsely modulate the background spiking probability of neurons in the network, allowing them to maintain locally asynchronous-irregular dynamics. These sparse-wave dynamics only become apparent when networks are modeled at sufficient scales, in particular hundreds of thousands of neurons over an area a few millimeters across (Fig 4B). Our custom software implementation allowed for the simulation of networks with approximately 100,000 to $1,000,000$ neurons, each with 3,000 synapses per cell, addressing in the largest networks over $60 \mathrm{~GB}$ of RAM. Importantly, while the cells in our network models have a large number of synapses per cell, the number of possible connection partners is high such that the network connectivity remains sparse. In these networks, the large number of synapses per cell allows the network to achieve self-sustained asynchronous-irregular activity when synaptic conductances are on the order observed in cortex ${ }^{34,55}$.

Unlike the outsized conductances typically used in smaller network simulations, the large scale networks simulated here enabled us to incorporate E/I synaptic strengths that were similar to those observed experimentally, leading to total E/l conductances on the order of the leak conductance ${ }^{20,56}$. This is advantageous because the larger conductances needed in smaller networks yield unrealistic coupling of spiking activity to synaptic fluctuations and shunt driving inputs ${ }^{57}$, as illustrated in the dense-wave model. By scaling our model to realistic neuronal densities on a spatial scale over several millimeters of cortex $\left(450,000\right.$ neurons over $16 \mathrm{~mm}^{2}$ in the case studied here), the sparse wave model sustains irregular activity, with strengths of individual synaptic inputs down to $0.5 \mathrm{nS}$ and $4 \mathrm{nS}$ for excitatory connections and inhibitory connections respectively. At these spatial scales and synaptic conductances, waves are present about $50 \%$ of the time, similar to what we find in neocortex, and the wavelengths closely approximate those we find in vivo. 
Traveling waves of neural activity in awake cortex have been observed under stimulusevoked $^{41,58,59}$, behavior-evoked ${ }^{60-62}$, and spontaneous conditions ${ }^{5}$. The fundamental neural circuit mechanism for these waves, however, had remained unclear. Our modeling results suggest that the spontaneous LFP fluctuations we observe traveling as waves in the cortex during active vision result from sparse waves of spiking activity traveling unmyelinated horizontal fibers. The sparse-wave model, which produces activity patterns consistent with the spiking activity observed in vivo, posits that these waves arise from the time delays inherent in communicating spikes across topographic connections within a cortical area. Further, observations from the model suggest that as these sparse waves traverse the massive recurrent connectivity within cortical areas ${ }^{2,35,63,64}$, they produce subthreshold shifts in the local E-I balance that, collectively, modulate cortical excitability. Thus, the model offers an explanation for our empirical finding that perceptual sensitivity varies over space and time depending on the alignment of wave phase ${ }^{5}$. Importantly, these traveling waves need not introduce correlated variability believed to harm perceptual sensitivity; instead, the sparse-wave state weakly modulates the background spiking probability in locally asynchronous-irregular neuron pools. Rather than a source of noise, as would be predicted if waves modulated activity akin to the dense-wave regime, the presence of these sparse waves can boost weak inputs that would otherwise have been imperceptible. These results indicate these traveling waves may be a network mechanism that can improve perceptual processing when aligned to the source of feedforward signals, without disrupting the computational benefits of the irregular spiking dynamics of individual neurons.

\section{Figures}

\section{Figure 1. Spontaneous network fluctuations are of similar magnitude to stimulus-evoked} responses. (a) Spike raster for repeated presentations $(N=40)$ of a high contrast $(10 \%$ Michelson contrast) drifting Gabor recorded from area MT of a fixating marmoset (stimulusonset, red line; mean response, blue line). A single trial LFP trace is plotted in gray, and the average LFP response is plotted in black. The relative power between baseline $(-100 \mathrm{~ms}$ to stimulus-onset) and evoked fluctuations (stimulus-onset to $100 \mathrm{~ms}$ ) significantly favored the evoked response (right panel; $\mathrm{N}=110$ trials; median $=2.78 \mathrm{~dB}, \mathrm{p}<1 \times 10^{-6}$, Wilcoxon rankedsum test). (b) Same as in (a), but for a low contrast stimulus ( $<2 \%$ Michelson contrast). The relative power between baseline and evoked LFP fluctuations was not statistically different from parity (median $=0.18 \mathrm{~dB}, \mathrm{p}=0.55$ Wilcoxon ranked-sum test). (c) Spontaneous LFP fluctuations 
recorded simultaneously from a spatially distributed multielectrode array were structured as traveling waves. (d) Histogram of spontaneous spike probability as a function of the Generalized Phase of the LFP during fixation. (e) The average evoked response to low contrast stimuli was stronger when a more excitable phase ( $\pm \pi \mathrm{rad})$ of a spontaneous traveling wave aligned with the retinotopic location of the target (green line) as compared to when a less excitable phase ( 0 rad) was aligned (purple line; shaded region S.E.M.; $p<0.01$ Wilcoxon ranked-sum test). Data for panels c-e modified from Davis et al. 2020 with permission.

\section{Figure 2. Topographically-connected networks produce structured fluctuations without} altering neuronal spiking dynamics. (a) Schematic diagram of a 2-D spiking network model with 80 percent excitatory (gray) and 20 percent inhibitory (blue) neurons wired with a random connection probability. (b) Spike rasters from 10000 excitatory neurons arranged by linear distance in the network. LFP fluctuations calculated from summed synaptic currents for a single $10 \times 10$ neuron pool is plotted in red. The mean spike rate across the same pool is shown in black. (c) Spatial organization of LFP amplitude for each neuron pool in the network plotted at one time. (d) Network schematic as in (a), but the network was topographically-connected with probabilities drawn from a Gaussian $(\sigma=400 \mu \mathrm{M})$, and activity had a distance dependent transmission delay $(0.2 \mathrm{~m} / \mathrm{s})$. (e) Spike rasters as in (b), but sparse fluctuations were apparent across the network. (f) Spatial LFP amplitude as in (c), but the LFP was heterogeneous across the network with topographic structure. (g) The distribution of single unit mean firing rates did not differ between the random (blue line) and topographic networks (purple line; $N=5000$ neurons; $p=0.68$, Kolmogorov-Smirnov test). (h) The distribution of single unit C.V. did not differ between the random and topographic networks ( $p=0.79$, Kolmogorov-Smirnov test). (i) Pairwise spike coherence did not differ between the random and topographic networks $(\mathrm{N}=1000$ random pairs; $\mathrm{Cl}$ test, $\mathrm{a}=0.05$; dotted lines $95 \% \mathrm{Cl}$ ).

Figure 3. Spontaneous topographic network fluctuations travel as waves. (a) Time series of simulated LFP activity from the topographically-connected network in Figure 2. Regional peaks and troughs moved coherently across the network as traveling waves. (b) Distribution of speeds for putative waves observed in the network. (c) Significant (white) and non-significant (black) wave values were defined as estimated wavelengths that exceeded the 99th percentile of the spatially shuffled wavelength distribution (47.67\% of network activity). (d) Cumulative distribution functions (CDFs) of the estimated LFP wavelengths (blue) and shuffle wavelengths (red, 99th percentile, red dashed line). (e) The randomly-connected network had few points that 
classify as traveling waves (1.12\% of network activity). (f). Wavelength CDFs for the randomlyconnected network and its shuffle.

Figure 4. Spontaneous traveling waves emerge in network regimes consistent with asynchronous-irregular dynamics. (a) The percentage of wave-like activity across ranges of excitatory and inhibitory conductances. Each pixel is one simulation of a network as described in figure 2, but with the excitatory and inhibitory conductances corresponding to its $\mathrm{x}$ and $\mathrm{y}$ coordinate, respectively. Networks consistent with asynchronous-irregular spiking dynamics fall within the black border. White pixels are networks that did not self sustain or had extremely low/high mean firing rates (FR $<1$ or $F R>25 \mathrm{sp} / \mathrm{sec}$ ). (b) Same as a, but the mean of the wavelength distribution is plotted. (c) The region of the parameter space that exhibited asynchronous-irregular activity grew with the size of the network (width $0.5 \mathrm{~mm}$ to $4 \mathrm{~mm}$ ). (d) The fraction of wave activity present across all self-sustained networks grew with the size of the simulated network (mean \pm SEM).

\section{Figure 5. Wavelength and speed distributions change with connection distance and} conduction velocity parameters. (a) CDFs for networks simulated across a range of Gaussian sigma values for the topographic connection probability. (b) Distributions of wave speeds across a range of networks simulated with different conduction velocities $(0.1$ to $0.6 \mathrm{~m} / \mathrm{s})$. (insert) The mean network speed plotted as a function of the simulated conduction velocity. (c) Waves (white pixels) were strongly present in a network with a mix of topographically (90\%) and randomly (10\%) connected projections and a range of conduction velocities $(0.1$ to $0.6 \mathrm{~m} / \mathrm{s})$. (d) CDFs for the simulation in (c) and its shuffle (blue and red respectively).

\section{Figure 6. Simulated network dynamics approximate dynamics observed in cortical} recordings of area MT. (a) Distribution of mean firing rates across simulated neurons in figure 2 (blue) and the distribution observed across single and multiunits recorded from area MT of awake, behaving marmosets (red). (b) The distributions of C.V. for simulated (blue) and recorded cortical data (red). (c) CDFs of wavelengths for the simulation in figure $5 \mathrm{c}$ (blue), its shuffle (yellow), and the observed distribution recorded in the cortex (red). Simulated wavelengths were similar to observed wavelengths in vivo after applying a smoothing kernel to approximate the estimated spatial integration of the LFP in electrophysiological recordings (250 $\mu \mathrm{M}$, gray dashed line). (d) Wave speed distributions for the simulation in figure $5 \mathrm{c}$ (blue), and recorded cortical data (red) covered a range consistent with axonal conduction velocities $(0.1$ to $0.6 \mathrm{~m} / \mathrm{s})$. 

modulate spike probability. (a) Membrane voltage for a simulated neuron in either the sparsewave network (black line) or dense-wave network (purple-line) calculated from the summed excitatory and inhibitory synaptic currents received by that neuron. Spiking activity occured when the voltage crosses the threshold $\left(\mathrm{V}_{\text {th }}\right.$ red line). The distribution of membrane potentials over the interval for the sparse and dense networks is plotted on the right. (b) The amplitude of the simulated LFP (blue line) and the relative level of excitatory and inhibitory currents (red line) over a 10x10 neuron pool were counterphase. (c) Scatter plot of LFP and E-I difference revealed a significant negative correlation ( $N=50000$ time points; Pearson's $r=-0.83$; $C l$ test, $\alpha=0.01$ ). (d) Spike-phase coupling was significant across networks in the asynchronous-irregular regime, and the degree of coupling was negatively correlated with the magnitude of synaptic conductances ( $\mathrm{N}=646$ simulations; Pearson's $r=-0.45 \pm 0.06, \mathrm{Cl}$ test, $\mathrm{a}=0.05$ ). (e) Histogram showing the fraction of spikes that occurred during each phase of the simulated LFP in the topographically-connected network. Spike probability was significantly modulated by LFP phase $\left(\mathrm{N}=22\right.$ resamples versus shuffle; circular-resultant $=0.15, \mathrm{p}<1 \times 10^{-4}$, Wilcoxon signed-rank test). (f) Same as in (e), but for recorded cortical data. Spike probability was similarly significantly modulated (circular-resultant $=0.16 ; \mathrm{N}=22$ recording sessions versus shuffle; $\mathrm{p}<1 \times 10^{-4}$, Wilcoxon signed-rank test). (g) The dense-wave network simulation had a significantly stronger spike-phase relationship ( $N=10$ resamples; circular-resultant $=0.43, p<1 \times 10^{-4}$, Wilcoxon ranked-sum test). network shunts. (a) The average firing rate across a neuron pool in the sparse-wave network model received a $50 \mathrm{~Hz}$ Poisson spike train input for $10 \mathrm{~ms}$ aligned either to a period of depolarization (blue shaded region) or hyperpolarization (red shaded region) as defined by the spike-LFP phase relationship. The blue and red lines are the mean evoked firing rate after receiving the spiking input in either the depolarized or hyperpolarized phase respectively (faint blue and red lines represent 20 individual trials). The black line is the firing rate of the neuron pool when no input was given. (b) Same as (a), but the inputs were delivered to the dense-wave

572 network. The evoked responses were much weaker as the network shunted the currents evoked 573 by the incoming spikes. (c) The response gain between the distributions of spontaneous and 574 evoked activity across 20 presentations of spiking input. In the sparse-wave network (left bars), 575 inputs during the depolarized state had larger relative responses as compared to inputs during 
600

601

602

603

604

605

606

607

608

609

the hyperpolarized state. Conversely, the dense-wave network (right bars) responses had higher gain during the hyperpolarized state, which was still significantly weaker than the depolarized state in the sparse-wave network $\left(4.25 \pm 0.11\right.$ v. $1.74 \pm 0.07 ; p<1 \times 10^{-7}$, Wilcoxon ranked-sum test).

\section{Supplemental Figures}

Figure S1. Dense spiking waves induce pairwise correlation while sparse spiking waves do not. (a) Spike raster for a 10000 neuron network simulation with topographic connections and delays. Dense spike participation in traveling waves produces large fluctuations in spiking activity (black line, pooled LFP example red line). (b) The spike-spike coherence for 1000 randomly selected pairs of neurons has a strong peak between $20-30 \mathrm{~Hz}$ (black line, dotted lines 95\% Cl) corresponding to the peak of the PSD for the example LFP (red line). (c) Spike probability is strongly coupled to the phase of the LFP.

\section{Figure S2. Randomly-connected spiking network model has weak spike-LFP phase} coupling. (a) Histogram showing the fraction of spikes that occurred during each phase of the LFP in the randomly connected network shown in Figure 2 (circular-resultant $=0.07$ ).

Table S1. Parameters for random, sparse-wave, and dense-wave network simulations

Table S2. Parameters for networks of various sizes

\section{Methods}

In vivo cortical recordings

One male (monkey $\mathrm{W}$ ) and one female (monkey $\mathrm{T}$ ) marmoset monkey (Callithrix jacchus) was surgically implanted with a headpost for head stabilization and eye tracking. The headpost contained a hollow chamber housing an Omnetics connector for a Utah array (Blackrock Microsystems), which was implanted in a $7 \times 10 \mathrm{~mm}$ craniotomy over area MT (stereotaxic coordinates $2 \mathrm{~mm}$ anterior, $12 \mathrm{~mm}$ dorsal). An 8x8 (64 channel, monkey W) and $9 \times 9$ with alternating channels removed (40 channel, monkey $\mathrm{T}$ ) Utah array was chronically implanted over area MT using a pneumatic inserter wand. The electrodes spacing was $400 \mu \mathrm{M}$ with a pitch depth 
610

611

612

613

614

615

616

617

618

619

620

621

622

623

624

625

626

627

628

629

630

631

632

633

634

635

636

637

638

639

640

641

of $1.5 \mathrm{~mm}$. The craniotomy was closed with Duraseal (Integra Life Sciences, monkey W) or Duragen (Integra Life Sciences, monkey T), and covered with a titanium mesh embedded in dental acrylic. All surgical procedures were performed with the monkeys under general anesthesia in an aseptic environment in compliance with $\mathrm{NIH}$ guidelines. All experimental methods were approved by the Institutional Animal Care and Use Committee (IACUC) of the Salk Institute for Biological Studies and conformed with NIH guidelines.

Marmosets were trained to enter a custom-built marmoset chair that was placed inside a faraday box with an LCD monitor (ASUS VG248QE) at a distance of $40 \mathrm{~cm}$. The monitor was set to a refresh rate of $100 \mathrm{~Hz}$ and gamma corrected with a mean gray luminance of 75 candelas $/ \mathrm{m}^{2}$. Electrode voltages were recorded from the Utah arrays using two Intan RHD2132 amplifiers connected to an Intan RHD2000 USB interface board. Data were sampled at $30 \mathrm{kHz}$ from all channels. The marmosets were headfixed by a headpost for all recordings. Eye position was measured with an IScan CCD infrared camera sampling eye position at $500 \mathrm{~Hz}$. Stimulus presentation and behavioral control was managed through MonkeyLogic in Matlab. Digital and analog signals were coordinated through National Instrument DAQ cards (NI PCI6621) and BNC breakout boxes (NI BNC2090A). Neural data was broken into two streams for offline processing of spikes (single-unit and multi-unit activity) and LFPs. Spike data was high-pass filtered at 500 $\mathrm{Hz}$ and candidate spike waveforms were defined as exceeding 4 standard deviations of a sliding 1 second window of ongoing voltage fluctuations. Artifacts were rejected if appearing synchronously (within $0.5 \mathrm{~ms}$ ) on over a quarter of all recorded channels. Segments of data (1.5 $\mathrm{ms}$ ) around the time of candidate spikes were selected for spike sorting using principal component analysis through the open source spike sorting software MClust in Matlab (A. David Redish, University of Minnesota). Sorted units were classified as single- or multi-units and single units were validated by the presence of a clear refractory period in the autocorrelogram. LFP data was low-pass filtered at $300 \mathrm{~Hz}$ and down-sampled to $1000 \mathrm{~Hz}$.

\section{Receptive Field Mapping}

Receptive fields were mapped through reverse correlation. The marmoset was trained to hold fixation on an image (marmoset face, 1 degree of visual angle (DVA) square) presented to the center of the LCD monitor. A drifting Gabor (2 degrees diameter, spatial frequency: 0.5 cycles per degree, temporal frequency 10 cycles per second) was presented at a random position on the monitor between 0-18 degrees in azimuth and -15 to 15 degrees in elevation, drifting in one of 8 possible directions for $200 \mathrm{~ms}$, after which it disappeared. After a random delay drawn from 
642

643

644

645

646

647

648

649

650

651

652

653

654

655

656

657

658

659

660

661

662

663

664

665

666

667

668

669

670

an exponential distribution (mean $40 \mathrm{~ms}$ ), a new probe appeared and the pattern repeated until the marmoset broke fixation (defined as an excursion of 1.5 degrees from fixation) or viewed 16 probes. The marmoset was given a juice reward proportional to the number of probes presented. The receptive field for each unit recorded on the array was estimated by calculating the spiketriggered average (STA) stimulus that evoked the maximal response:

$$
S T A=\frac{1}{N} \sum_{i}^{N} x_{i} y_{i}
$$

The STA is the sum of probe location $x_{i}$ weighted by the spike count $y_{i}$ within the time bin 40 to $200 \mathrm{~ms}$ after probe onset, normalized by the number of all recorded spikes $N$. From the location of estimated receptive fields on each spiking channel, and the known topography of area MT in the marmoset ${ }^{65}$, we estimated the relative position of each recording array in marmoset cortex (Fig. 1a). We excluded from the analysis the upper half of monkey W's array as the recordings did not appear to be in area MT.

\section{Behavioral Task}

The marmosets were trained to saccade to a marmoset face to initiate each trial, after which the face turned into a fixation point (0.15 DVA). The marmosets held fixation on the fixation point (1.5 degree tolerance) for a minimum duration (400 ms monkey W, 300 ms monkey $\mathrm{T}$ ) awaiting the appearance of a drifting Gabor (4 DVA diameter which reliably produced evoked responses on 1-2 electrodes; spatial frequency, 0.5 cycles per degree; temporal frequency, 10 cycles per second, drifting in one of up to 8 possible directions). Spontaneous data were analyzed from the period of fixation preceding the appearance of a target. Early fixation breaks (defined by the excursion of the eye position from the fixation window) were excluded from analysis. The target only appeared if fixation was held for an additional random duration drawn from an exponential distribution (mean $200 \mathrm{~ms}$ ) to generate a flat hazard function.

\section{Computational Simulations}

The model consists of $N$ leaky integrate-and-fire (LIF) neurons, with $N_{e}=0.8 N$ excitatory units and $N_{i}=0.2 N$ inhibitory. The membrane potential $V$ of the $i$ th unit evolves in time according to the equation: 
673 where $C_{m}$ is the membrane capacitance, $g_{L}$ is the leak conductance, $E_{L}$ is the resting membrane 674 potential, $g_{\{e, i\}}$ are the time-dependent synaptic conductances, respectively, and $E_{\{e, i\}}$ are the 675 reversal potentials for excitatory and inhibitory synaptic transmission. When the membrane 676 potential $V_{i}$ exceeds threshold $V_{t}$, the model obeys the following spike and reset conditions:

677

$678 \quad V_{i} \rightarrow V_{r}$

$679 g_{\{e, i\}, j} \rightarrow g_{\{e, i\}, j}+w_{\{e, i\}} \forall j \in[1, K]$

680

681 where $V_{r}$ is the reset potential, $w_{\{e, i\}}$ are the excitatory and inhibitory synaptic weights, 682 respectively, and $K$ is the number of postsynaptic targets of neuron $i$. Following the initial 683 conductance increase caused by an incoming spike, the excitatory and inhibitory synaptic 684 conductances follow exponential decay:

$\tau_{\{e, i\}} \dot{g}_{\{e, i\}}=-g_{\{e, i\}}$

687

where ${ }^{\tau}\{e, i\}$ are the excitatory and inhibitory synaptic time constants.

689

690

Network connectivity and axonal conduction delays

691

692

We studied either spiking network models with unstructured, random connectivity (random networks, Fig. 2a) or topographic, locally random connectivity (topographic networks, Fig. 2d, for parameters see Table S1). Neurons in the random network were connected to $K$ post-synaptic targets chosen with uniform probability. Axonal delays $(\tau)$ in the random network were short and homogeneous $(300 \mu \mathrm{s})$. In the topographic network, connections were chosen to $K$ post-synaptic targets with probability decaying as a Gaussian with distance from the sending neuron, with spatial width $\sigma_{s}$ of $400 \mu \mathrm{m}$ (except where the effect of this parameter is studied systematically in 
704

705

706

707

708

709

710

711

712

713

714

715

716

717

718

719

720

721

722

723

724

725

726

727

728

729

730

731

732

733

734

735

where $\tau_{i j}$ is the delay from neuron $i$ to neuron $j, \tau_{s}$ is the delay caused by synaptic vesicle release,

$d_{i j}$ is the Euclidean distance between $i$ and $j$, and $v_{c}$ is the axonal conduction speed. All distances were calculated taking 2D periodic boundary conditions into account, effectively wrapping the network onto a toroidal topology ${ }^{55,66}$.

\section{Self-sustained activity}

Instead of initializing self-sustained activity through a "kick" of external Poisson input spikes $^{33,34,37}$, which may induce trace activity correlations, we recorded the state variables of a self-sustained network, including membrane potential $(V)$ and conductance $\left(g_{\{e, i\}}\right)$, after a long period (10 seconds) of simulated self-sustained activity. Taking these distributions as a steady state, we then used the Gaussian approximation (mean and variance) to initialize the membrane potentials and conductances with randomly drawn values in the simulations thereafter. After starting the simulation with these initial conditions, networks with approximately balanced excitation and inhibition exhibit self-sustained, irregular spiking activity. Each simulation ran for 1.2 seconds, from which we eliminated the first $200 \mathrm{~ms}$ from our analysis in case of residual initialization artifacts.

\section{Spike train statistics and the asynchronous-irregular regime}

To characterize basic spike train statistics, we randomly selected 5,000 neurons in the simulation and measured the mean firing rate, coefficient of variation (C.V., defined as the ratio of the standard variation of the interspike interval to the mean for each neuron that has a minimum of 3 spikes over the simulation window), and the average pairwise correlation (average Pearson correlation between spike trains smoothed with a 100 ms window for 1,000 randomly selected pairs). To prevent longer simulations with high firing rate during our parallel runs, networks that produced mean firing rates over $50 \mathrm{sp} / \mathrm{s}$ had an early exit condition. Simulations were classified as asynchronous-irregular if the mean firing rate across all simulated units was greater than 1 and less than $25 \mathrm{sp} / \mathrm{s}$; the mean C.V. across all units was greater than 0.7 and less than 1.4, and the average pairwise cross-correlation was greater than -0.1 and less than 0.1 .

\section{Pairwise Spike Coherence}


Pairwise spike coherence was calculated using multitaper methods as described previously ${ }^{67}$. The $1000 \mathrm{~ms}$ of simulation time was broken into $500 \mathrm{~ms}$ epochs, stepping $125 \mathrm{~ms}$ to cover the full period. The DC component of each unit's spike train was removed, and tapered with a single Slepian taper, giving an effective smoothing of $2.5 \mathrm{~Hz}$ for the $500 \mathrm{~ms}$ data windows (NW $=1, \mathrm{~K}$ $=1$ ). To control for differences in firing rate among units, randomly chosen spikes were eliminated in order to equate the total number of spikes in each paired spike train. This procedure was repeated 10 times and the results were averaged to produce the single paired coherence. Coherence was then computed across 1000 randomly selected pairs of units, and normalized by the pooled power spectrum. Confidence intervals were evaluated using a jack knife procedure leaving out individual pairs of units.

\section{NETSIM software}

Simulations were generated using a specialized program called NETSIM, which is approximately 1,500 lines of $\mathrm{C}$ code (available at http://mullerlab.github.io). Equations in the model were integrated using the forward Euler method with a timestep of $0.1 \mathrm{~ms}$. Simulation results were additionally point-checked with shorter timesteps throughout. Random numbers were generated using a $\mathrm{C}$ implementation of the ISAAC algorithm ${ }^{68}$ (Tom Bartol, personal communication). To verify the numerical integration in this program, we confirmed the network displayed the correct firing rate for unconnected LIF neurons with varying DC-current injections. We also verified simulations under a simple feed-forward network topology to confirm the accuracy of the simulations. Additionally, to ensure reproducibility of our computational simulations ${ }^{69}$, we compared results from NETSIM and Brian2 at specific points in the $\left(g_{e}, g_{i}\right)$ parameter space for the balanced random network model, verifying that the mean firing rate, C.V., and crosscorrelation were in agreement between the two simulators.

\section{Network Parameter Scans}

In order to identify the excitatory and inhibitory synaptic conductance weights that produced self sustained and asynchronous-irregular activity, we simulated networks with 50 values of $g_{e}$ ranging from 0.1 to $5 \mathrm{nS}$ and $g_{i}$ ranging from 1 to $50 \mathrm{nS}$ for a total of 2,500 simulations (Table S2). In order to determine the effect of network scale on the range of these 2,500 simulations that produced self sustained and asynchronous-irregular activity, we repeated these simulations 5 times with varying parameters of network size, neuron number, and connections per neuron 
(Table S2). The number of neurons per network size was chosen to maintain a density of 28,125 neurons per $\mathrm{mm}^{2}$. The number of connections was chosen to maintain the density of connections within the Gaussian used to assign connections across network sizes. For larger networks (>2 $\mathrm{mm}$ ) the connection number did not grow with the size of the network, as 99 percent of the connections occur within 3 S.D. of the Gaussian $(\sigma=400 \mu \mathrm{M})$. In order to run these simulations across all combinations of network size and conductance parameters, we utilized the Extreme Science Engineering Discovery Environment (XSEDE) Comet cluster at the San Diego SuperComputer (SDSC) center at UC San Diego. Data analysis for these simulations was also performed on the Comet cluster running MATLAB. Circular variables were analyzed using the Circular Statistics Toolbox.

\section{Calculation of LFP estimate}

We utilized a previously developed proxy for the LFP generated by a network of point LIF neurons, which was systematically developed from a spatially extended model ${ }^{38}$. The LFP estimate $\lambda(t)$ is computed as a weighted sum of the excitatory and inhibitory synaptic currents $I_{c}$ and $I_{i}$ across $m$ excitatory cells in each $10 \times 10$ neuron pool:

$$
\lambda(t)=\sum_{j}^{m} I_{e, j}(t-\tau)-\alpha \sum_{j}^{m} I_{i, j}(t)
$$

where $\tau=6 \mathrm{~ms}$ and $\alpha=1.65$. This LFP proxy is then computed for each $10 \times 10$ neuron pool across the two-dimensional network. This proxy was initially computed independently for each neuron pool, excluding effects from mixing of the LFP signal across space. We note that excluding these effects is a conservative step, as the addition of the mixing across space would only increase traveling waves in the LFP. Further, we note that our results do not depend critically on the choice of LFP proxy and our conclusions are unchanged when analyzing the mean membrane potentials or excitatory synaptic conductances.

In order to compare the properties of waves in our model, where LFP signals are independent across space, from waves recorded from the cortex, where electrodes pool signals across a volume approximately $250 \mu \mathrm{M}$ in radius ${ }^{29,70}$, we convolved the LFP with a 2D Gaussian kernel (with a spatial standard deviation of 4 LFP bins, corresponding to a radius of $272 \mu \mathrm{M}$ ) before further analysis. 
801

802

803

804

805

806

807

808

809

810

811

812

813

814

815

816

817

818

819

820

821

822

823

824

825

826

827

828

829

830

831

832

833

\section{Analysis of spatiotemporal dynamics}

To analyze spatiotemporal dynamics in the population activity produced by the spiking network model, we used a technique we recently developed for wideband analysis of non-stationary data. Briefly, for each real-valued time series $\lambda_{x, y}(t) \forall x \in\left[1, N_{c}\right], y \in\left[1, N_{r}\right]$, where $N_{c}$ and $N_{r}$ are the number of columns and rows, respectively, we compute the generalized phase (GP) $\phi_{x, y}$ at each point using the corrected analytic signal representation introduced in recent work ${ }^{5}$. We next calculated the gradient of GP $\vec{g}_{x, y}(t)$ at each moment in time:

$$
\vec{g}_{x, y}(t)=-\nabla \phi_{x, y}(t)
$$

For the spatial gradient, derivatives are taken across the two dimensions of space and are approximated by the appropriate forward and centered finite differences. As in previous work, phase derivatives were implemented as multiplications in the complex plane ${ }^{41,71}$, so that unwrapping phase across the two dimensions of the network was not necessary. Wavelength is the reciprocal of the phase gradient magnitude at each point in space and time. Wave speed was computed as the ratio of instantaneous frequency to phase gradient magnitude, as in previous work ${ }^{60}$.

\section{Calculation of response gain}

In order to quantify the sensitivity of the sparse and dense-wave network regimes to incoming stimulation, we first identified depolarized and hyperpolarized states from the LFP of a $10 \times 10$ neuron pool defined by the spike-phase bins that generated the maximum or minimum spiking probability respectively for each network regime. We then applied feed-forward stimulation of 50 $\mathrm{Hz}$ Poisson spiking input to 100 synapses for each neuron within the $10 \times 10$ neuron pool for 10 ms, aligned to the depolarized or hyperpolarized phase in the network. This process was repeated across 20 trials, yielding a distribution of evoked responses. The same random seed was used to construct the networks across each trial, so that the simulations were identical up to the point of stimulation. We calculated the ratio of the evoked responses to the spontaneous response in the absence of any stimulation at the defined time point.

Acknowledgments: The authors would like to thank Katie Williams, Sean Adams, and Mat LeBlanc for their contributions to this project, in addition to the San Diego Supercomputer Center for their support for this project. The authors would also like to thank Tom Bartol for discussions 
on the software implementation. Funding: Gatsby Charitable Foundation, the Fiona and Sanjay Jha Chair in Neuroscience, the Canadian Institute for Health Research, the Swartz Foundation, NIH grants R01-EY028723, T32 EY020503-06, T32 MH020002-16A, and BrainsCAN at Western University through the Canada First Research Excellence Fund (CFREF).

Author Contributions: Conceptualization, Z.W.D., G.B.B., C.F., T.D., T.J.S., J.H.R., L.M.; Data Curation, Z.W.D., G.B.B. C.F., T.D., L.M.; Formal Analysis, Z.W.D., G.B.B., C.F., T.D., L.M.; Funding Acquisition, Z.W.D., T.J.S., J.H.R., L.M.; Investigation, Z.W.D., G.B.B., L.M.; Methodology, Z.W.D., G.B.B., C.F., T.S., J.H.R., L.M.; Supervision, T.J.S., J.H.R., L.M.; Visualization, Z.W.D., G.B.B., L.M.; Writing - original draft, Z.W.D., G.B.B., C.F., T.D. J.H.R., L.M.; Writing - review and editing, Z.W.D., G.B.B., T.J.S., J.H.R., L.M.

Competing Interests: Authors declare no competing interests.

Materials \& Correspondence: Correspondence and requests for material should be addressed to J.H.R and L.M.

Data Availability: The data that support the findings of this study are available from the corresponding author upon reasonable request.

Code Availability: An open-source code repository for all methods is available on http://mullerlab.github.io.

\section{References}

1. Destexhe, A. \& Rudolph-Lilith, M. Neuronal Noise: (Springer US, 2012).

2. Destexhe, A., Rudolph, M. \& Paré, D. The high-conductance state of neocortical neurons in vivo. Nat. Rev. Neurosci. 4, 739-751 (2003).

3. Destexhe, A. Intracellular and computational evidence for a dominant role of internal network activity in cortical computations. Curr. Opin. Neurobiol. 21, 717-725 (2011).

4. Girard, P., Hupé, J. M. \& Bullier, J. Feedforward and feedback connections between areas V1 and V2 of the monkey have similar rapid conduction velocities. J. Neurophysiol. 85, 1328-1331 (2001).

5. Davis, Z. W., Muller, L., Martinez-Trujillo, J., Sejnowski, T. \& Reynolds, J. H. Spontaneous travelling cortical waves gate perception in behaving primates. Nature (2020) doi:10.1038/s41586-020-2802-y. 
6. Brunel, N. Dynamics of sparsely connected networks of excitatory and inhibitory spiking neurons. J. Comput. Neurosci. 8, 183-208 (2000).

7. Renart, A. et al. The asynchronous state in cortical circuits. Science 327, 587-590 (2010).

8. Nandy, A., Nassi, J. J., Jadi, M. P. \& Reynolds, J. Optogenetically induced low-frequency correlations impair perception. Elife 8, (2019).

9. Croner, L. J., Purpura, K. \& Kaplan, E. Response variability in retinal ganglion cells of primates. Proc. Natl. Acad. Sci. U. S. A. 90, 8128-8130 (1993).

10. Mainen, Z. F. \& Sejnowski, T. J. Reliability of spike timing in neocortical neurons. Science 268, 1503-1506 (1995).

11. Shadlen, M. N. \& Newsome, W. T. Noise, neural codes and cortical organization. Curr. Opin. Neurobiol. 4, 569-579 (1994).

12. Arieli, A., Sterkin, A., Grinvald, A. \& Aertsen, A. Dynamics of ongoing activity: explanation of the large variability in evoked cortical responses. Science 273, 1868-1871 (1996).

13. Tsodyks, M., Kenet, T., Grinvald, A. \& Arieli, A. Linking spontaneous activity of single cortical neurons and the underlying functional architecture. Science 286, 1943-1946 (1999).

14. Kenet, T., Bibitchkov, D., Tsodyks, M., Grinvald, A. \& Arieli, A. Spontaneously emerging cortical representations of visual attributes. Nature 425, 954-956 (2003).

15. Han, F., Caporale, N. \& Dan, Y. Reverberation of recent visual experience in spontaneous cortical waves. Neuron 60, 321-327 (2008).

16. Ermentrout, G. B., Bard Ermentrout, G. \& Kleinfeld, D. Traveling Electrical Waves in Cortex. Neuron vol. 29 33-44 (2001).

17. Destexhe, A., Contreras, D. \& Steriade, M. Spatiotemporal analysis of local field potentials and unit discharges in cat cerebral cortex during natural wake and sleep states. J. Neurosci. 19, 4595-4608 (1999).

18. Churchland, M. M. et al. Stimulus onset quenches neural variability: a widespread cortical phenomenon. Nat. Neurosci. 13, 369-378 (2010).

19. Sato, T. K., Nauhaus, I. \& Carandini, M. Traveling waves in visual cortex. Neuron 75, 218 229 (2012).

20. El Boustani, S., Pospischil, M., Rudolph-Lilith, M. \& Destexhe, A. Activated cortical states: experiments, analyses and models. J. Physiol. Paris 101, 99-109 (2007).

21. Destexhe, A. \& Contreras, D. Neuronal computations with stochastic network states. Science 314, 85-90 (2006). 
22. Fiser, J., Chiu, C. \& Weliky, M. Small modulation of ongoing cortical dynamics by sensory input during natural vision. Nature 431, 573-578 (2004).

23. Luczak, A., Bartho, P. \& Harris, K. D. Gating of sensory input by spontaneous cortical activity. J. Neurosci. 33, 1684-1695 (2013).

24. Gutnisky, D. A., Beaman, C. B., Lew, S. E. \& Dragoi, V. Spontaneous Fluctuations in Visual Cortical Responses Influence Population Coding Accuracy. Cereb. Cortex (2016) doi:10.1093/cercor/bhv312.

25. Destexhe, A., Rudolph, M., Fellous, J. M. \& Sejnowski, T. J. Fluctuating synaptic conductances recreate in vivo-like activity in neocortical neurons. Neuroscience (2001).

26. Chance, F. S., Abbott, L. F. \& Reyes, A. D. Gain modulation from background synaptic input. Neuron 35, 773-782 (2002).

27. Tomko, G. J. \& Crapper, D. R. Neuronal variability: non-stationary responses to identical visual stimuli. Brain Res. 79, 405-418 (1974).

28. Shadlen, M. N. \& Newsome, W. T. The variable discharge of cortical neurons: implications for connectivity, computation, and information coding. J. Neurosci. 18, 3870-3896 (1998).

29. Katzner, S. et al. Local origin of field potentials in visual cortex. Neuron 61, 35-41 (2009).

30. Lindén, H. et al. Modeling the spatial reach of the LFP. Neuron 72, 859-872 (2011).

31. Abbott, L. F. \& van Vreeswijk C. Asynchronous states in networks of pulse-coupled oscillators. Phys. Rev. E Stat. Phys. Plasmas Fluids Relat. Interdiscip. Topics 48, 14831490 (1993).

32. Softky, W. R. \& Koch, C. The highly irregular firing of cortical cells is inconsistent with temporal integration of random EPSPs. J. Neurosci. 13, 334-350 (1993).

33. Vogels, T. P. \& Abbott, L. F. Signal propagation and logic gating in networks of integrateand-fire neurons. J. Neurosci. 25, 10786-10795 (2005).

34. Kumar, A., Schrader, S., Aertsen, A. \& Rotter, S. The high-conductance state of cortical networks. Neural Comput. 20, 1-43 (2008).

35. Binzegger, T., Douglas, R. J. \& Martin, K. A. C. A quantitative map of the circuit of cat primary visual cortex. J. Neurosci. 24, 8441-8453 (2004).

36. Atapour, N. et al. Neuronal Distribution Across the Cerebral Cortex of the Marmoset Monkey (Callithrix jacchus). Cereb. Cortex 29, 3836-3863 (2019).

37. Destexhe, A. Self-sustained asynchronous irregular states and Up-Down states in thalamic, cortical and thalamocortical networks of nonlinear integrate-and-fire neurons. $J$. Comput. Neurosci. 27, 493-506 (2009). 
38. Mazzoni, A. et al. Computing the Local Field Potential (LFP) from Integrate-and-Fire Network Models. PLoS Comput. Biol. 11, e1004584 (2015).

39. Hellwig, B. A quantitative analysis of the local connectivity between pyramidal neurons in layers 2/3 of the rat visual cortex. Biol. Cybern. 82, 111-121 (2000).

40. Levy, R. B. \& Reyes, A. D. Spatial profile of excitatory and inhibitory synaptic connectivity in mouse primary auditory cortex. J. Neurosci. 32, 5609-5619 (2012).

41. Muller, L., Reynaud, A., Chavane, F. \& Destexhe, A. The stimulus-evoked population response in visual cortex of awake monkey is a propagating wave. Nat. Commun. 5, 3675 (2014).

42. Muller, L. et al. Rotating waves during human sleep spindles organize global patterns of activity that repeat precisely through the night. Elife 5, (2016).

43. Stettler, D. D., Das, A., Bennett, J. \& Gilbert, C. D. Lateral connectivity and contextual interactions in macaque primary visual cortex. Neuron 36, 739-750 (2002).

44. Angelucci, A. et al. Circuits for local and global signal integration in primary visual cortex. J. Neurosci. 22, 8633-8646 (2002).

45. Bringuier, V., Chavane, F., Glaeser, L. \& Frégnac, Y. Horizontal propagation of visual activity in the synaptic integration field of area 17 neurons. Science 283, 695-699 (1999).

46. Lewis, D. A. Horizontal Synaptic Connections in Monkey Prefrontal Cortex. Cerebral Cortex Jan 10, 82-92 (2000).

47. Destexhe, A. Oscillations, complex spatiotemporal behavior, and information transport in networks of excitatory and inhibitory neurons. Phys. Rev. E Stat. Phys. Plasmas Fluids Relat. Interdiscip. Topics 50, 1594-1606 (1994).

48. Zerlaut, Y. \& Destexhe, A. Enhanced Responsiveness and Low-Level Awareness in Stochastic Network States. Neuron 94, 1002-1009 (2017).

49. Izhikevich, E. M. Polychronization: computation with spikes. Neural Comput. 18, 245-282 (2006).

50. Ermentrout, B. Neural networks as spatio-temporal pattern-forming systems. Rep. Prog. Phys. 61, 353-430 (1998).

51. Osan, R. \& Ermentrout, B. Two dimensional synaptically generated traveling waves in a theta-neuron neural network. Neurocomputing 38, 789-795 (2001).

52. Gong, P. \& van Leeuwen, C. Distributed dynamical computation in neural circuits with propagating coherent activity patterns. PLoS Comput. Biol. 5, e1000611 (2009).

53. Keane, A. \& Gong, P. Propagating waves can explain irregular neural dynamics. J. Neurosci. 35, 1591-1605 (2015). 
54. Huang, C. et al. Circuit Models of Low-Dimensional Shared Variability in Cortical Networks. Neuron 101, 337-348.e4 (2019).

55. Kumar, A., Rotter, S. \& Aertsen, A. Conditions for propagating synchronous spiking and asynchronous firing rates in a cortical network model. J. Neurosci. 28, 5268-5280 (2008).

56. Rudolph, M., Pospischil, M., Timofeev, I. \& Destexhe, A. Inhibition determines membrane potential dynamics and controls action potential generation in awake and sleeping cat cortex. J. Neurosci. 27, 5280-5290 (2007).

57. Morrison, A., Aertsen, A. \& Diesmann, M. Spike-Timing-Dependent Plasticity in Balanced Random Networks. Neural Computation vol. 19 1437-1467 (2007).

58. Yang, Z., Heeger, D. J., Blake, R. \& Seidemann, E. Long-range traveling waves of activity triggered by local dichoptic stimulation in V1 of behaving monkeys. J. Neurophysiol. 113, 277-294 (2015).

59. Chemla, S. et al. Suppressive Traveling Waves Shape Representations of Illusory Motion in Primary Visual Cortex of Awake Primate. J. Neurosci. 39, 4282-4298 (2019).

60. Rubino, D., Robbins, K. A. \& Hatsopoulos, N. G. Propagating waves mediate information transfer in the motor cortex. Nat. Neurosci. 9, 1549-1557 (2006).

61. Zanos, T. P., Mineault, P. J., Nasiotis, K. T., Guitton, D. \& Pack, C. C. A sensorimotor role for traveling waves in primate visual cortex. Neuron 85, 615-627 (2015).

62. Balasubramanian, K. et al. Propagating Motor Cortical Dynamics Facilitate Movement Initiation. Neuron 106, 526-536.e4 (2020).

63. Braitenberg, V. \& Schüz, A. Cortex: Statistics and Geometry of Neuronal Connectivity. (Springer, 1998).

64. Markov, N. T. et al. Weight consistency specifies regularities of macaque cortical networks. Cereb. Cortex 21, 1254-1272 (2011).

65. Rosa, M. G. P. \& Elston, G. N. Visuotopic organisation and neuronal response selectivity for direction of motion in visual areas of the caudal temporal lobe of the marmoset monkey (Callithrix jacchus): middle temporal area, middle temporal crescent, and surrounding cortex. J. Comp. Neurol. 393, 505-527 (1998).

66. Mehring, C., Hehl, U., Kubo, M., Diesmann, M. \& Aertsen, A. Activity dynamics and propagation of synchronous spiking in locally connected random networks. Biol. Cybern. 88, 395-408 (2003).

67. Mitchell, J. F., Sundberg, K. A. \& Reynolds, J. H. Spatial attention decorrelates intrinsic activity fluctuations in macaque area V4. Neuron 63, 879-888 (2009). 
996 68. Jenkins, R. J. ISAAC. in Fast Software Encryption 41-49 (Springer Berlin Heidelberg, 997 1996).

998 69. Davison, A. P. et al. PyNN: A Common Interface for Neuronal Network Simulators. Front. $999 \quad$ Neuroinform. 2, 11 (2008).

1000 70. Buzsáki, G., Anastassiou, C. A. \& Koch, C. The origin of extracellular fields and currents1001 EEG, ECoG, LFP and spikes. Nat. Rev. Neurosci. 13, 407-420 (2012).

1002 71. Feldman, M. Hilbert transform in vibration analysis. Mech. Syst. Signal Process. 25, 735$1003802(2011 / 4)$. 


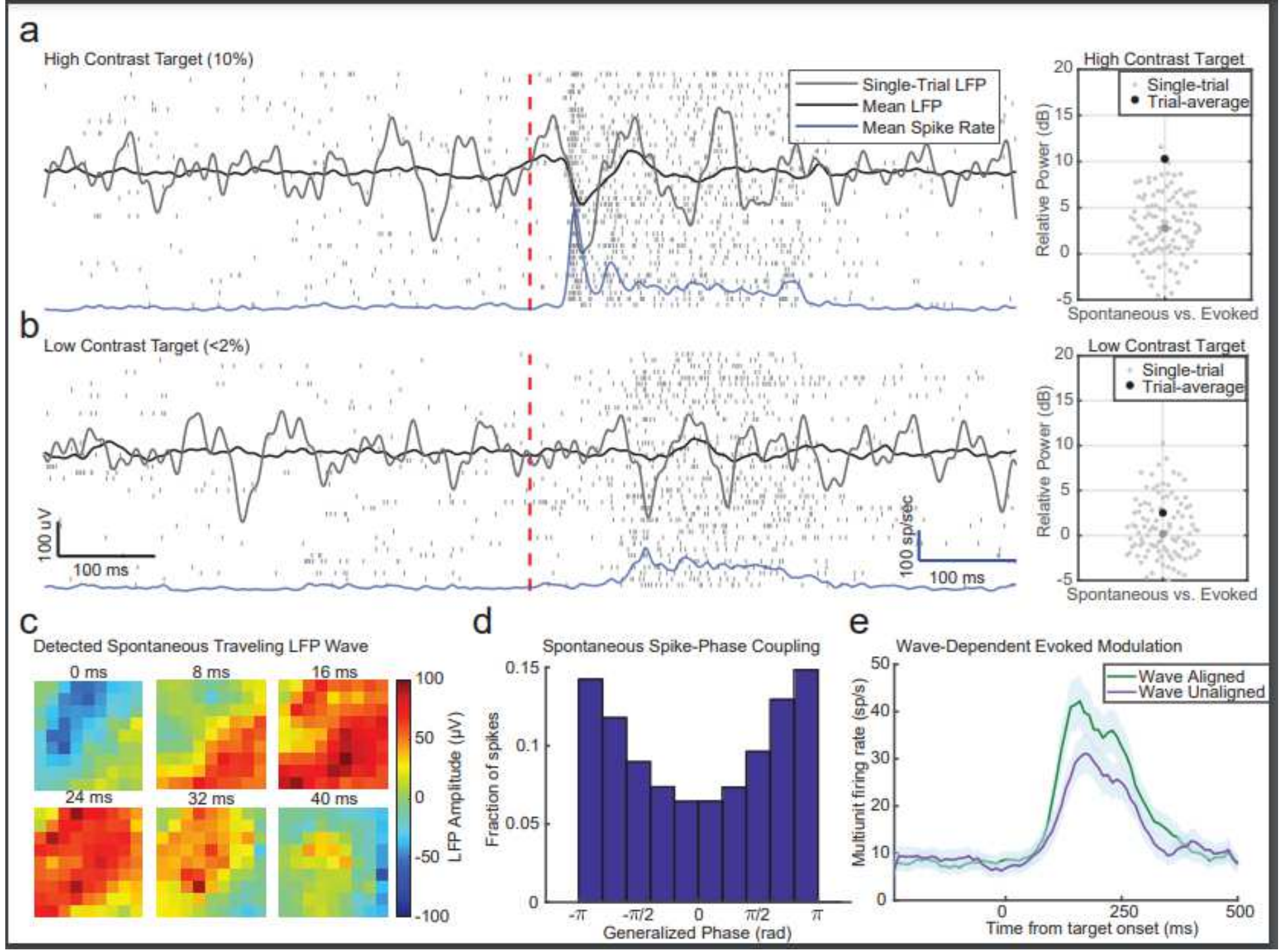

Figure 1

[Please see the manuscript file to view the figure caption.] 


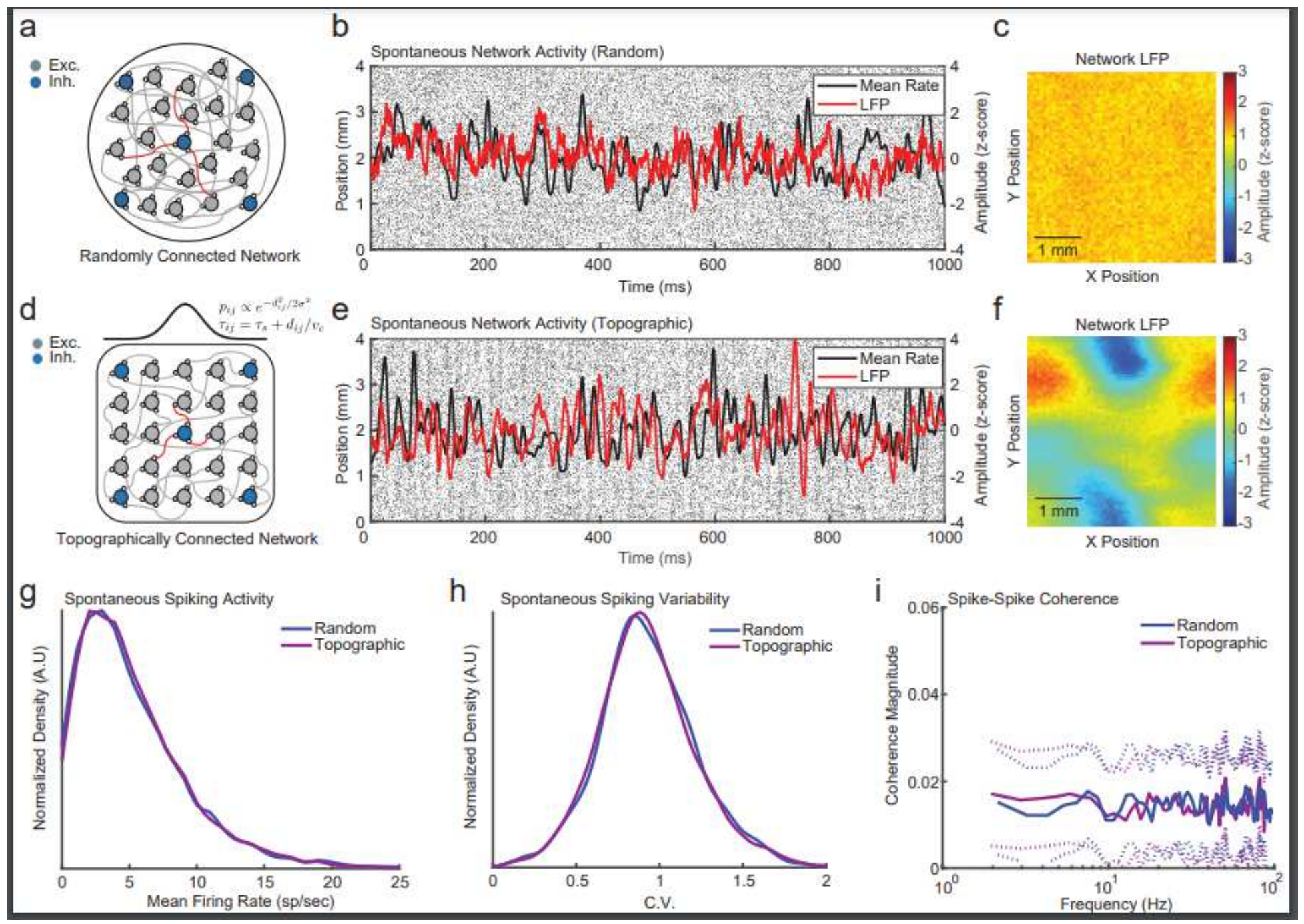

Figure 2

[Please see the manuscript file to view the figure caption.] 


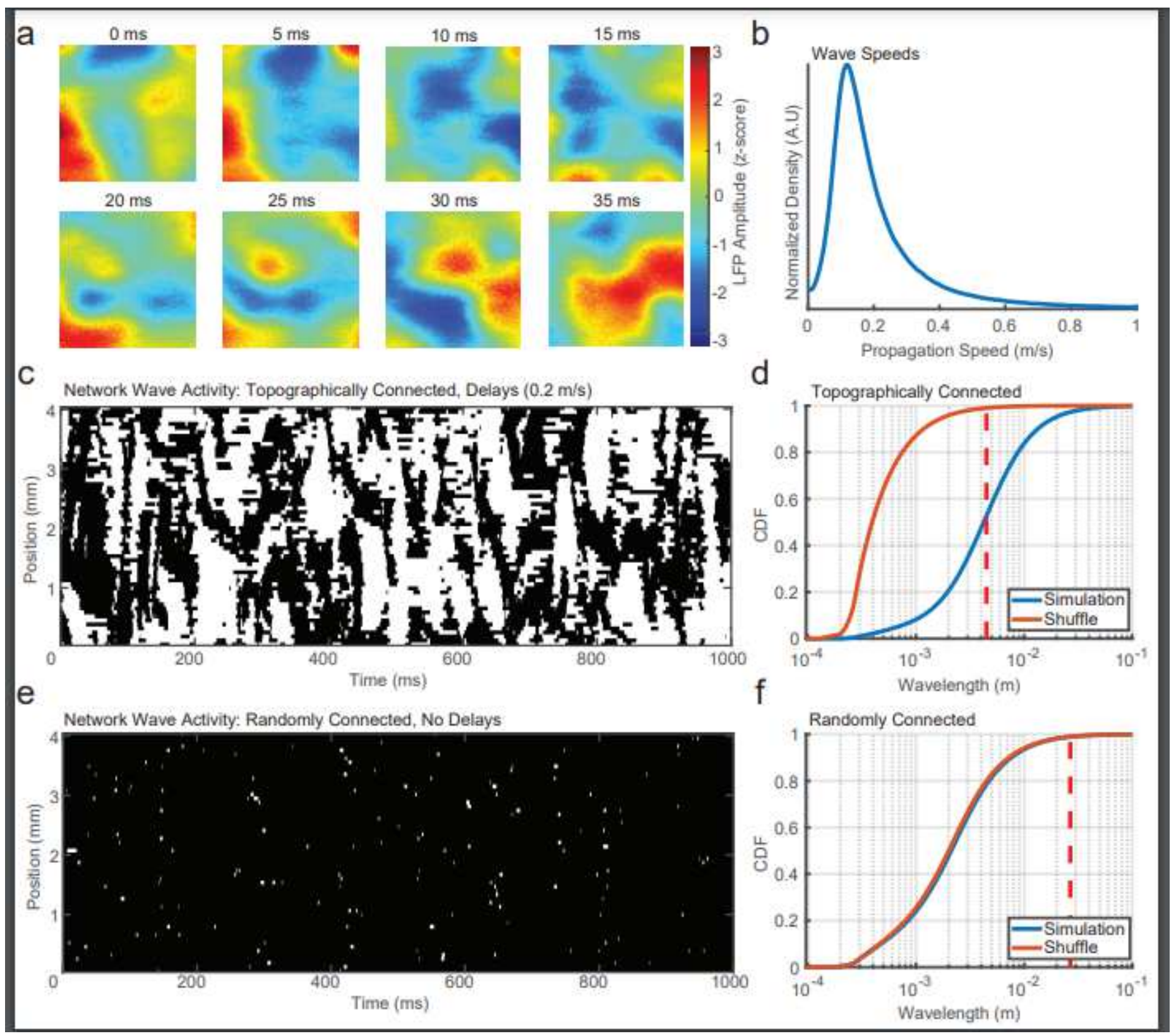

Figure 3

[Please see the manuscript file to view the figure caption.] 


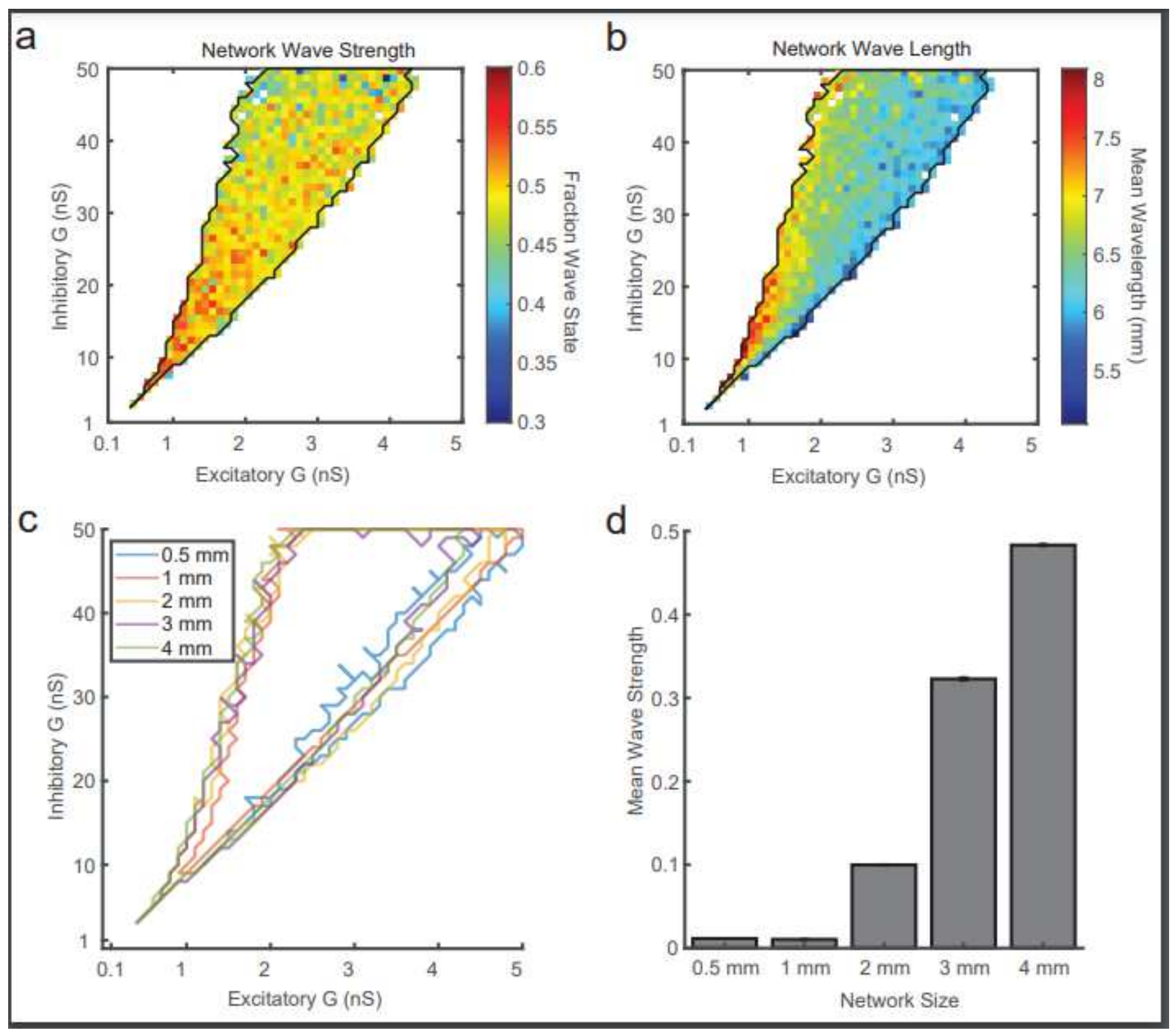

Figure 4

[Please see the manuscript file to view the figure caption.] 


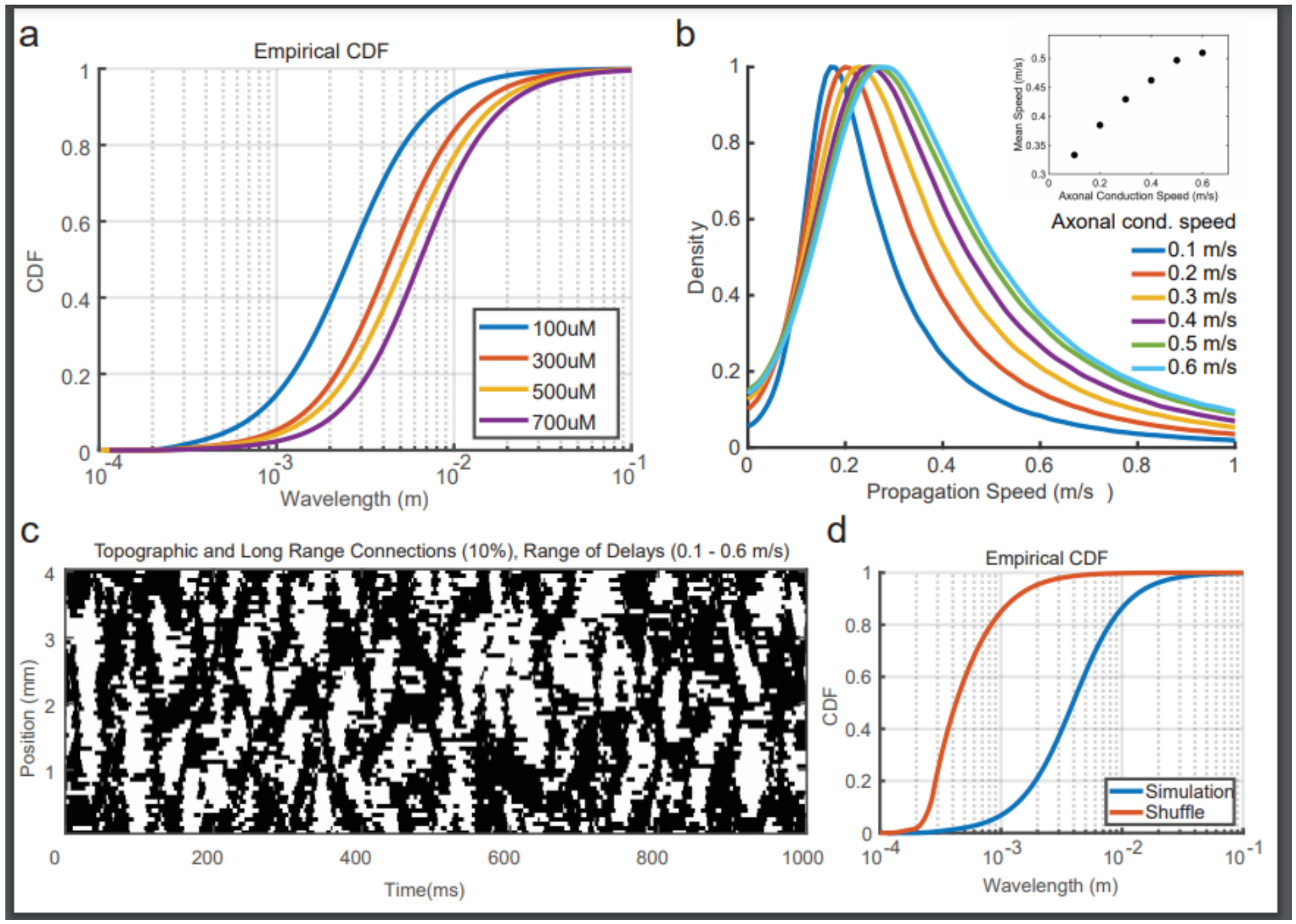

Figure 5

[Please see the manuscript file to view the figure caption.] 


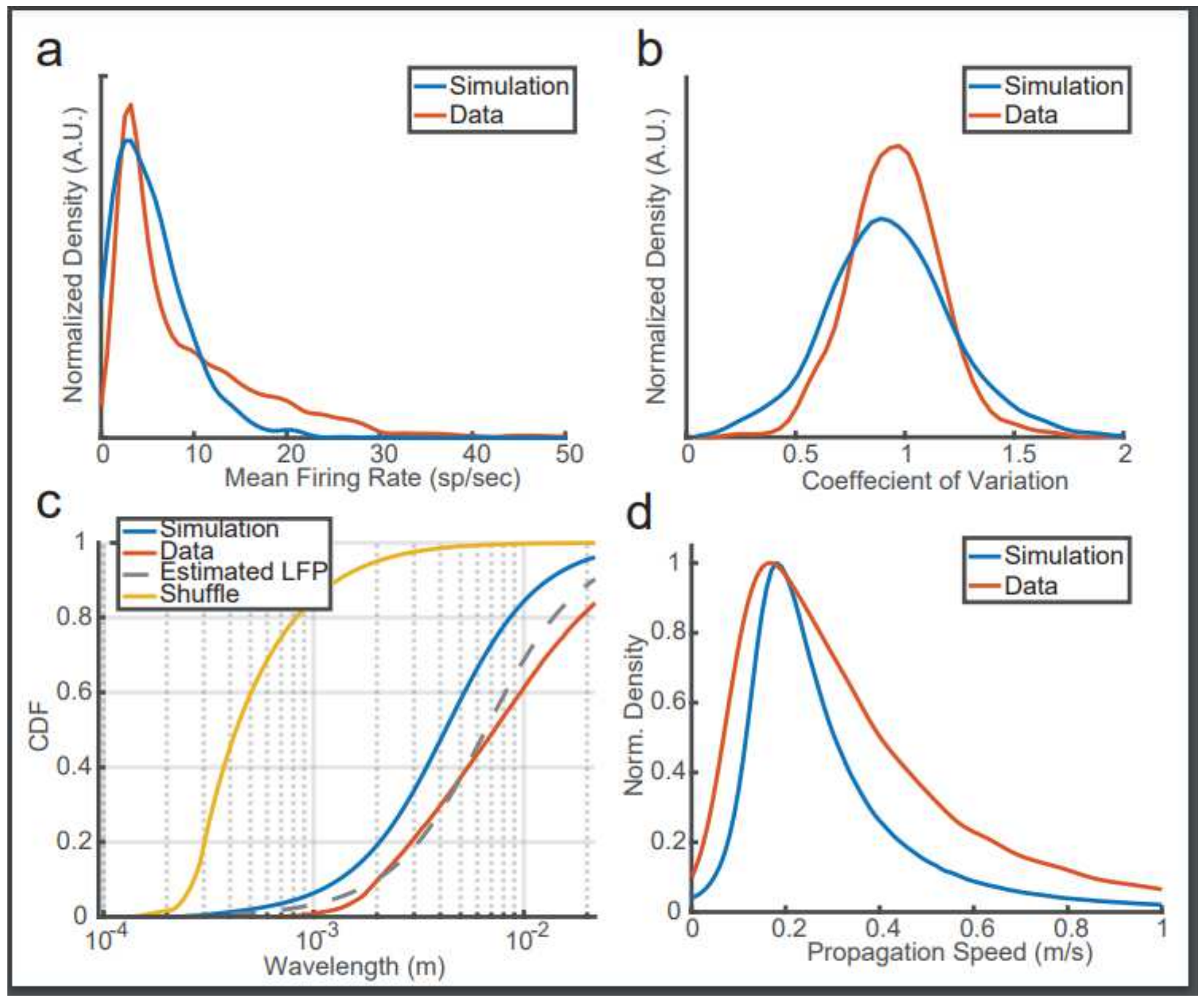

Figure 6

[Please see the manuscript file to view the figure caption.] 


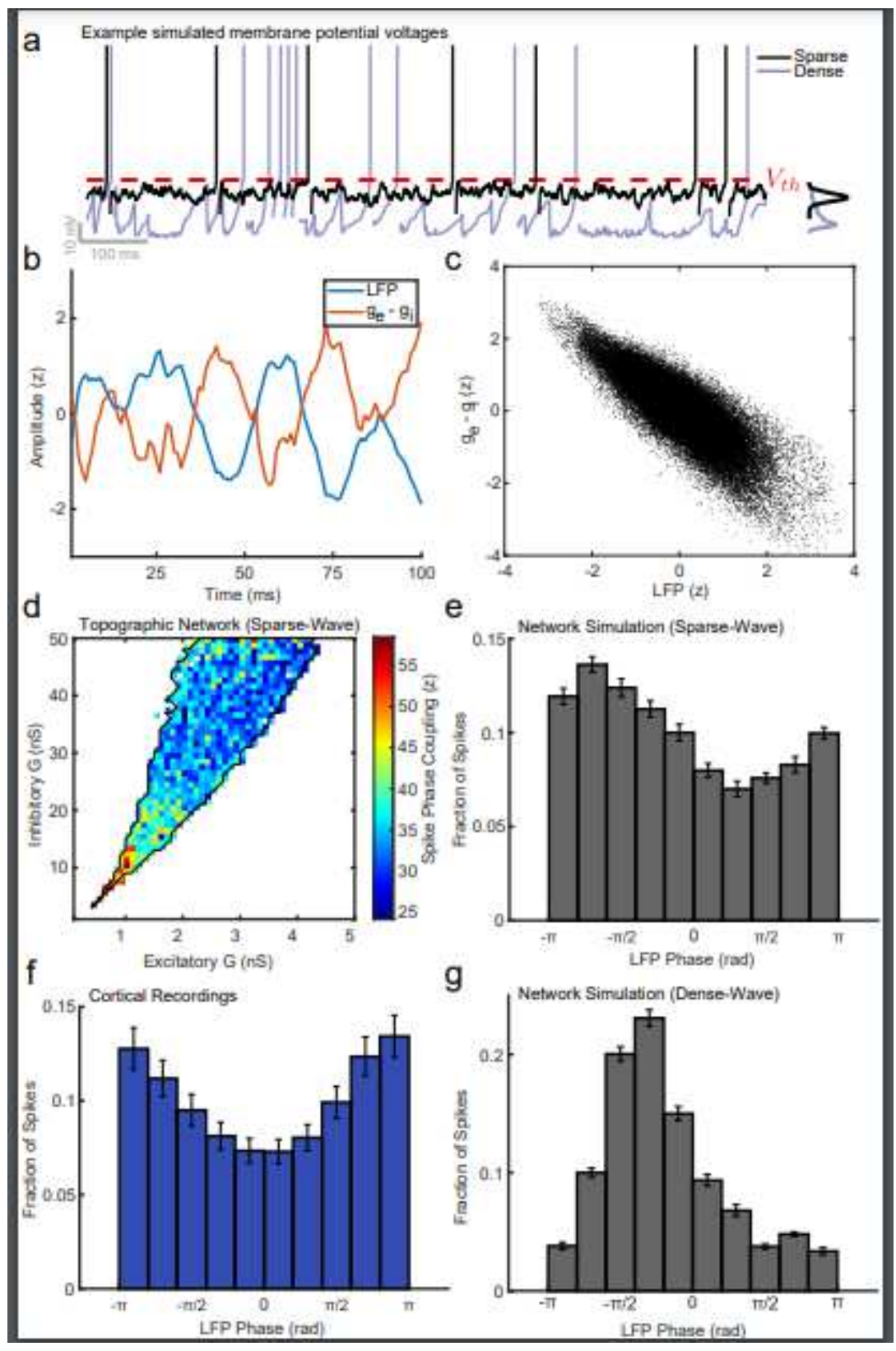

Figure 7

[Please see the manuscript file to view the figure caption.]

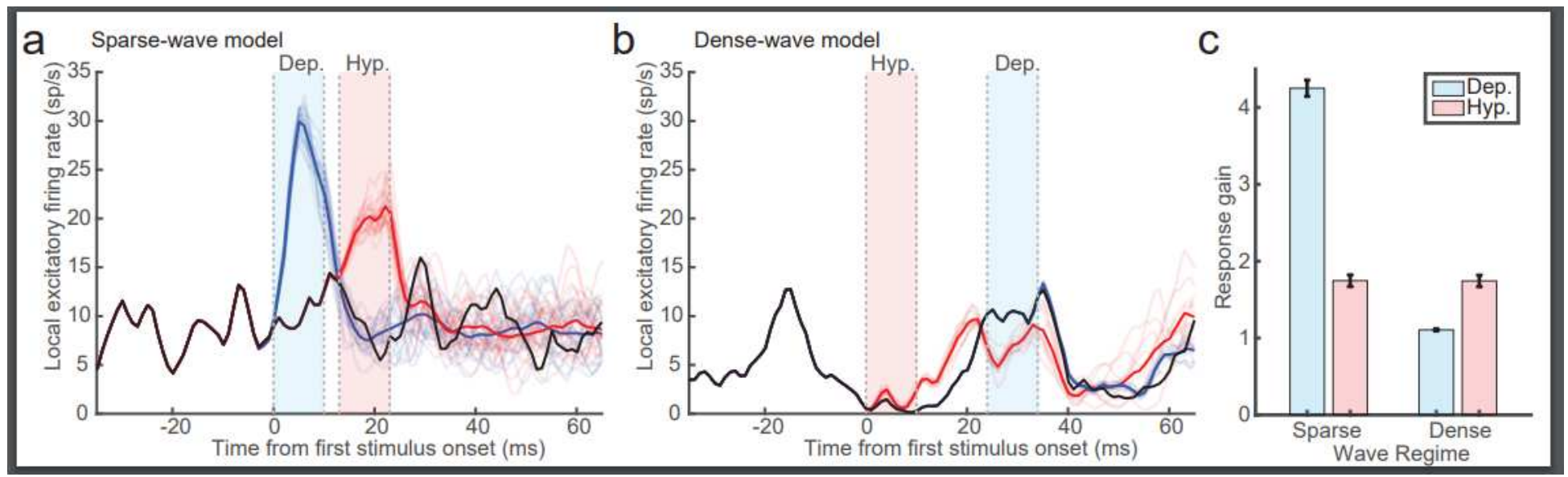


Figure 8

[Please see the manuscript file to view the figure caption.]

\section{Supplementary Files}

This is a list of supplementary files associated with this preprint. Click to download.

- tableS1.pdf

- tables2.pdf

- figureS2.pdf

- FigureS1.pdf 